\title{
Complex Ginzburg-Landau equations with dynamic boundary conditions
}

\author{
Wellington José Corrêa ${ }^{\mathrm{a}}$, Türker Özsarl ${ }^{\mathrm{b}, *}$ \\ a Academic Department of Mathematics, Federal Technological University of Paraná, Campuses Campo \\ Mourão, 87301-899, Campo Mourão, PR, Brazil \\ ${ }^{\mathrm{b}}$ Department of Mathematics, Izmir Institute of Technology, Izmir, Turkey
}

\section{A R T I C L E I N F O}

\section{Article history:}

Received 20 July 2017

Received in revised form 27

November 2017

Accepted 1 December 2017

Available online 20 December 2017

\section{Keywords:}

Dynamic boundary conditions

Complex Ginzburg-Landau equations

Inviscid limits

\begin{abstract}
A B S T R A C T
The initial-dynamic boundary value problem (idbvp) for the complex GinzburgLandau equation (CGLE) on bounded domains of $\mathbb{R}^{N}$ is studied by converting the given mathematical model into a Wentzell initial-boundary value problem (ibvp). First, the corresponding linear homogeneous idbvp is considered. Secondly, the forced linear idbvp with both interior and boundary forcings is studied. Then, the nonlinear idbvp with Lipschitz nonlinearity in the interior and monotone nonlinearity on the boundary is analyzed. The local well-posedness of the idbvp for the CGLE with power type nonlinearities is obtained via a contraction mapping argument. Global well-posedness for strong solutions is shown. Global existence and uniqueness of weak solutions are proven. Smoothing effect of the corresponding evolution operator is proved. This helps to get better well-posedness results than the known results on idbvp for nonlinear Schrödinger equations (NLS). An interesting result of this paper is proving that solutions of NLS subject to dynamic boundary conditions can be obtained as inviscid limits of the solutions of the CGLE subject to same type of boundary conditions. Finally, long time behavior of solutions is characterized and exponential decay rates are obtained at the energy level by using control theoretic tools.
\end{abstract}

○ 2017 Elsevier Ltd. All rights reserved.

\section{Introduction}

This article is devoted to the analysis of the initial-dynamic boundary value problem (idbvp) for the complex Ginzburg-Landau equation (CGLE):

$$
\begin{cases}u_{t}-(\lambda+i \alpha) \Delta u+f(u)=0 & \text { in } \Omega \times \mathbb{R}_{+}, \\ \frac{\partial u}{\partial \nu}=-g\left(u_{t}\right) & \text { on } \Gamma_{1} \times \mathbb{R}_{+}, \\ u=0 & \text { on } \Gamma_{0} \times \mathbb{R}_{+}, \\ u(0)=u_{0} & \text { in } \Omega .\end{cases}
$$

\footnotetext{
* Corresponding author.

E-mail addresses: wcorrea@utfpr.edu.br (W.J. Corrêa), turkerozsari@iyte.edu.tr (T. Özsarı).
} 
In (1.1), $\Omega \subset \mathbb{R}^{N}$ is a bounded regular domain with boundary $\Gamma$, which is the union of $\Gamma_{0}$ and $\Gamma_{1}$, two nonempty, non-intersecting, connected $(n-1)$-dimensional manifolds. $u=u(x, t)$ is a complex valued function that denotes the complex oscillation amplitude. $f(u)$ will be defined either as the usual power type nonlinearity $f(u)=(\kappa+i \beta)|u|^{p-1} u-\gamma u$ (Sections 6-10) or as an appropriate Lipschitz function (Section 5). Here, $\beta \in \mathbb{R}$ and $\alpha \in \mathbb{R}$ are the (nonlinear) frequency and (linear) dispersion parameters, respectively. Without loss of generality, $\alpha$ can be taken as positive. Therefore we will also assume $\alpha>0$ throughout the text. $\beta$ can have both signs except when we discuss global solutions with power type nonlinearities, where it will be assumed to be positive. $\frac{\partial u}{\partial \nu}$ denotes the unit outward normal derivative. $p \geq 2$ is the source power index. Other parameters satisfy $\lambda, \kappa>0, \gamma \in \mathbb{R} . g: \mathbb{C} \rightarrow \mathbb{C}$ is a complex valued function which is either taken as identity (Sections 2-4, 6-10) or as a monotone function (Section 5) satisfying suitable growth conditions to be specified later in Assumption 2.1.

CGLE is a fundamental model in mathematical physics to describe near-critical instability waves, such as a reaction diffusion system near a Hopf-bifurcation. Concrete applications of this equation include nonlinear waves, second-order phase transitions, superconductivity, superfluidity, Bose-Einstein condensation, and liquid crystals. See [1] and the references therein for an overview of several phenomena described by the CGLE.

CGLE simultaneously generalizes the nonlinear heat and nonlinear Schrödinger equations (NLS), both of which can be obtained in the limit as the parameter pairs $(\alpha, \beta)$ and $(\lambda, \kappa)$ tend to zero, respectively. Therefore, it is natural to expect that CGLE carries some of the characteristics of the nonlinear heat equation and NLS. The latter two types of equations have been studied to some extent under dynamic boundary conditions. However, there has been no such progress for the CGLE. Most models assumed ideal set-ups neglecting possible linear and nonlinear interior-boundary interactions. See for example [2-12], and [13] for existence and non-existence results on the CGLE in the case of the whole space or domains with homogeneous or periodic boundary conditions. There are only a few results on the CGLE under nonhomogeneous boundary conditions [14-18]. The NLS subject to inhomogeneous or nonlinear boundary conditions, which can be considered a limiting case of the CGLE, took much more attention in recent years; see for example [19-28], [29-32], and [33].

Recently, [34] studied the defocusing cubic Schrödinger equation with dynamic boundary conditions on a bounded domain $\Omega \subset \mathbb{R}^{N}$ with smooth boundary for $N=2,3$. The model considered in [34] was the special case of the problem (1.1) where $\alpha=\beta=1$ and $\lambda=\kappa=\gamma=0$. In this work, the authors obtained the local well-posedness of strong $\left(H^{2}\right)$ solutions for $N=2,3$ and global well-posedness of strong solutions for $N=2$. In addition, the existence (without uniqueness) of weak $\left(H^{1}\right)$ solutions was obtained for $N=2,3$. Moreover, it was proven that the energy of the weak solutions satisfies a uniform decay rate estimate under appropriate monotonicity conditions imposed on the nonlinear term appearing in the dynamic boundary conditions.

The key idea in [34] is replacing the given dynamic boundary condition with an equivalent boundary condition, which is obtained by replacing $u_{t}$ on the boundary with the Laplacian and other terms coming from the main equation. This enables one to obtain the generation of a semigroup in an appropriate topology. The idea of using a boundary condition which involves the trace of the Laplacian comes from Venttsel's work [35]. In his paper, Venttsel was interested in finding the most general boundary condition which restricts the closure of a given elliptic operator to the infinitesimal generator of a semigroup of positive contraction operators on the Banach space of continuous functions over a regular compact region [35]. The result of this work was the discovery of the generalized Venttsel (more commonly "Wentzell") boundary condition $a \Delta u+b \frac{\partial u}{\partial \nu}+c u=0$ on $\Gamma$, which provided the desired property for $a>0, b, c \geq 0$.

Physically, this boundary condition can be considered as a (damped) harmonic oscillator acting at each point on the boundary. In the case of the heat equation, this means that the boundary can act as a heat source or sink depending on the physical situation. These boundary conditions also arise naturally in the 
study of the wave equation. In particular, generalized Wentzell boundary conditions can be thought of as a closed subclass of acoustic boundary conditions. The well-posedness of Wentzell problems for the heat equation was proved on spaces of the form $X_{p}=L^{p}(\Omega) \cup L^{p}(\Gamma)$. See for example [36], where the Wentzell problem is treated as a coupled system of two PDEs: one on the interior and one on the boundary. Regarding Wentzell boundary conditions, it is also worth mentioning the papers [34,37], and [36-39] on heat and wave equations.

We are interested in studying three main problems for the CGLE model considered in (1.1): (i) wellposedness (ii) inviscid limits (iii) long-time behavior. However, there are some challenges:

(i) The method used on the heat equation [36] fails when $\alpha \neq 0$.

(ii) The linear version of (1.1) does not generate a semigroup at the $L^{2}$-level. Since $L^{2}(\Omega)$ is not the natural underlying topology for problem (1.1) for semigroup generation, one cannot directly employ the monotone operator theory [10] (a common tool to treat CGLE) since the operator $B u=|u|^{p} u$ is $m$-accretive only in $L^{2}(\Omega)$, but not for example in $H^{1}(\Omega)$.

(iii) There is no control of the $L^{2}(\Omega)$-norm of the solution due to the presence of a non-standard boundary condition. This is a major drawback for treating the nonlinear terms via Gagliardo-Nirenberg type estimates. Focusing problems become particularly difficult even under smallness assumptions on the power of the nonlinearities or initial datum.

One can overcome some of the difficulties above by using the method presented in [34]. However, the additional terms in (1.1) compared to the NLS equation make the analysis more subtle.

The intrinsic regularizing feature of the Ginzburg-Landau operator provides more flexibility from the point of regularity compared to NLS [34]. Let us mention a few differences between the two studies:

(i) We prove that solutions of the CGLE possess better interior regularity than the solutions of the NLS. This result verifies the natural smoothing effect of the semigroup generator (see for example Theorem 2.3, Corollary 2.1, Lemma 7.1, and Theorem 2.7). This latter property is due to the fact that the parabolic effect in the CGLE is not present in the Schrödinger dynamics.

(ii) In order to prove the local existence of strong solutions we construct a special complete metric space ( Lemma 6.2) whose elements are compatible at time $t=0$ with the initial datum $u_{0}$. We show that the solution operator maps this complete metric space (actually a suitably chosen closed ball in it) onto itself in a contractive manner. Due to our construction of this complete metric space, the initial datum satisfies the necessary compatibility condition at each step of the contraction argument, which allows us to use the linear non-homogeneous theory.

(iii) Controlling the $H^{1}(\Omega)$ norm of the solutions is trivial for the defocusing NLS. In the case of the CGLE, the energy functional (see (7.1) and (7.2)) involves other terms such as $\frac{1}{p+1}(\alpha \kappa+\beta \lambda)\|u\|_{L^{p+1}\left(\Gamma_{1}\right)}^{p+1}$, $\alpha \lambda \int_{0}^{t}\|\Delta u\|_{L^{2}(\Omega)}^{2} d s$, and $\int_{0}^{t}\|u(s)\|_{L^{2 p}(\Omega)}^{2 p} d s$. In order to achieve a similar type of control of the energy for the solutions of CGLE, we make an assumption on the sign of the frequency parameter.

(iv) The smoothing effect is also utilized here in obtaining global well-posedness results for a wider range of parameters $N$ and $p$. The fact that $\lambda>0$ in the CGLE helps us to obtain better control estimates compared to the case of the nonlinear Schrödinger equation. [34] obtains global well-posedness of strong solutions only in dimension $N=2$ and $p=3$. We are able to improve this result in the context of the CGLE and obtain global well-posedness of strong solutions for $p \geq 2$ if $N=1 ; p \in[2,5]$ if $N=2$; and $p \in\left[2, \frac{11}{3}\right]$ if $N=3$.

(v) We show that solutions of the idbvp for NLS can be obtained as inviscid limits of solutions of the idbvp for CGLE as the parameter pair $(\lambda, \kappa) \rightarrow 0$. This gives one another approach to study NLS with dynamic boundary conditions. Inviscid limits and convergence to the NLS for the CGLE at different topological levels have been previously studied in the case of the whole space, periodic or 
homogeneous boundary conditions (see e.g., [2,40], and [41]). We verify this property at the $H^{1}$-level under dynamic boundary conditions. Moreover, we give a rather non-standard regularity in time for $u_{t}$ when $u \in L^{\infty}\left(0, T ; V \equiv H_{\Gamma_{0}}^{1}(\Omega)\right)$ is the weak solution of the idbvp for the NLS. More precisely, we prove that $u_{t}$ indeed belongs to $L^{2}\left(0, T ; V^{\prime}\right)$ as opposed to $L^{\infty}\left(0, T ; V^{\prime}\right)$ (the common space for the classical models).

The rest of the paper is organized as follows:

(i) Section 2 is devoted to the description of notations, assumptions, and statements of the main results.

(ii) In Sections 3 and 4, we discuss the well-posedness of the corresponding linear homogeneous and nonhomogeneous systems.

(iii) In Section 5, we study the Lipschitz perturbations of the linear equation with monotone boundary conditions.

(iv) In Section 6, we obtain the local well-posedness of strong solutions for (1.1).

(v) In Section 7, we study the global strong solutions.

(vi) In Section 8, we discuss the existence and uniqueness of weak solutions.

(vii) In Section 9, we prove that solutions of the idbvp for the NLS can be obtained as inviscid limits of the solutions of the idbvp for the CGLE.

(viii) Finally, in Section 10, we study the long time behavior of solutions and obtain exponential decay rates by using a special multiplier, which is now a classical tool in the control theory of PDEs.

\section{Notation and main results}

We consider the space $L^{2}(\Omega)$ of complex valued functions on $\Omega$ endowed with the inner product

$$
(y, z)_{L^{2}(\Omega)}=\int_{\Omega} y(x) \bar{z}(x) d x
$$

and the induced norm

$$
\|y\|_{L^{2}(\Omega)}^{2}=(y, y)_{L^{2}(\Omega)} .
$$

We also consider the Sobolev space $H^{1}(\Omega)$ endowed with the scalar product

$$
(y, z)_{H^{1}(\Omega)}=(y, z)_{L^{2}(\Omega)}+(\nabla y, \nabla z)_{L^{2}(\Omega)} .
$$

We will observe later that the natural underlying space for problem (1.1) is

$$
V=\left\{u \in H^{1}(\Omega) ; u=0 \text { on } \Gamma_{0}\right\}
$$

instead of the common $L^{2}(\Omega)$. $V^{\prime}$ will denote the dual space of $V$.

Since $\Gamma_{0} \neq \emptyset$, due to Poincaré's inequality, we can consider the space $V$ endowed with the norm induced by the scalar product

$$
(y, z)_{V}=(\nabla y, \nabla z)_{L^{2}(\Omega)}, \forall y, z \in V .
$$

The norm $\|y\|_{V} \equiv\|\nabla y\|_{L^{2}(\Omega)}$ is equivalent to the usual norm of $H^{1}(\Omega)$.

In what follows, we define the operator $A$ as the sum of the Schrödinger and heat operators, that is,

$$
A \equiv(\lambda+i \alpha) \Delta
$$


with the domain given by

$$
D(A) \equiv\left\{y \in V, \Delta y \in V, \frac{\partial y}{\partial \nu}=-(\lambda+i \alpha) \Delta y \text { on } \Gamma_{1}\right\} .
$$

In the above definition, $\left.\Delta\right|_{\Gamma_{1}}$ should be interpreted as the restriction of the Laplacian from the interior to the boundary, which is well-defined according to the Sobolev trace theory since $\Delta y \in V$ for $y \in D(A)$.

We first study the linear idbvp corresponding to (1.1) with $g \equiv i d$ :

$$
\begin{cases}u_{t}=(\lambda+i \alpha) \Delta u & \text { in } \Omega \times(0, \infty), \\ u=0 & \text { on } \Gamma_{0} \times(0, \infty), \\ \frac{\partial u}{\partial \nu}=-u_{t} & \text { on } \Gamma_{1} \times(0, \infty), \\ u(0)=u_{0} & \text { in } \Omega .\end{cases}
$$

The operator $A$ given in (2.4) recasts the idbvp (2.6) as the following Wentzell initial-boundary value problem (ibvp):

$$
\begin{cases}u_{t}=(\lambda+i \alpha) \Delta u & \text { in } \Omega \times(0, \infty), \\ u=0 & \text { on } \Gamma_{0} \times(0, \infty), \\ \frac{\partial u}{\partial \nu}=-(\lambda+i \alpha) \Delta u & \text { on } \Gamma_{1} \times(0, \infty), \\ u(0)=u_{0} & \text { in } \Omega .\end{cases}
$$

In abstract operator theoretic form, we can rewrite (2.7) alternatively as

$$
\dot{u}=A u, u(0)=u_{0} .
$$

Using the above reformulation of problem (2.6), we are able to prove the following well-posedness result.

Theorem 2.1 (Linear Homogeneous Problem I). The operator $(A, D(A))$ generates a strongly continuous semigroup of contractions on $V$.

Going back to the idbvp (2.6), one can restate the above theorem as follows.

Theorem 2.2 (Linear Homogeneous Problem II). Let $u_{0} \in V$. Then there exists a unique solution $u \in C([0, \infty) ; V)$ to problem $(2.6)$.

In order to deal with the nonlinear problem (1.1), we first study the following non-homogeneous model:

$$
\begin{cases}u_{t}-(\lambda+i \alpha) \Delta u=f & \text { in } \Omega \times(0, \infty), \\ u=0 & \text { on } \Gamma_{0} \times(0, \infty), \\ \frac{\partial u}{\partial \nu}=-u_{t} & \text { on } \Gamma_{1} \times(0, \infty), \\ u(0)=u_{0} & \text { in } \Omega .\end{cases}
$$

The above idbvp, as in the linear homogeneous case, can be treated as a Wentzell ibvp. Indeed, it is considered as a special case of the more general Wentzell problem below:

$$
\begin{cases}u_{t}-(\lambda+i \alpha) \Delta u=f & \text { in } \Omega \times(0, \infty) \\ u=0 & \text { on } \Gamma_{0} \times(0, \infty) \\ \frac{\partial u}{\partial \nu}=-(\lambda+i \alpha) \Delta u+g & \text { on } \Gamma_{1} \times(0, \infty) \\ u(0)=u_{0} & \text { in } \Omega\end{cases}
$$

with a given internal forcing term $f$ and boundary input $g$.

We prove the following result for problem (2.9). 
Theorem 2.3 (Linear Nonhomogeneous Problem I). Let $f \in L^{1}(0, \infty ; V)$ and $g \in L^{2}\left(0, \infty ; L^{2}\left(\Gamma_{1}\right)\right)$. Then for each $u_{0} \in V$ there exists a unique solution $u \in C([0, \infty) ; V)$ to $(2.9)$. Moreover, $\Delta u \in L^{2}\left(0, \infty ; L^{2}(\Omega)\right)$ and the following "hidden" trace regularity holds true:

$$
\frac{\partial u}{\partial \nu} \in L^{2}\left(0, \infty, L^{2}\left(\Gamma_{1}\right)\right) .
$$

The Wentzell ibvp in (2.9) can be formally identified with the non-homogeneous idbvp (2.8) with the special choice $g \equiv-\left.f\right|_{\Gamma_{1}}$.

Corollary 2.1 (Linear Nonhomogeneous Problem II). Let $f \in L^{2}(0, \infty ; V)$. Then for each $u_{0} \in V$ there exists a unique solution $u \in C([0, \infty) ; V)$ to $(2.8)$. Moreover, $\left.u_{t}\right|_{\Gamma_{1}} \in L^{2}\left(0, \infty ; L^{2}\left(\Gamma_{1}\right)\right), u_{t} \in L^{2}\left(0, \infty ; L^{2}(\Omega)\right)$ and $u \in L^{2}\left(0, \infty ; H^{2}(\Omega)\right)$.

Remark 2.1. Due to the smoothing component of the operator $A$ given in (2.4), the solution obtained here carries more regularity than the solution obtained in the case of the Schrödinger equation (compare Theorem 2.3 and Corollary 2.1 with [34, Theorem 1.4 and Corollary 1.5]).

We can extend the linear theory to include Lipschitz perturbations (both on the interior and on the boundary) and nonlinear dynamic boundary feedback.

$$
\begin{cases}u_{t}=(\lambda+i \alpha) \Delta u+f(u) & \text { in } \Omega \times(0, \infty), \\ u=0 & \text { on } \Gamma_{0} \times(0, \infty), \\ \frac{\partial u}{\partial \nu}=-g\left(u_{t}\right) & \text { on } \Gamma_{0} \times(0, \infty), \\ u(0)=u_{0} & \text { in } \Omega .\end{cases}
$$

Here, $g(z)$ is assumed to satisfy the following conditions:

Assumption 2.1. Assume that $g(z)$ is a continuous function on $\mathbb{C}$ such that both $g(z)$ and its inverse $g^{-1}(z)$ satisfy:

(i) $\operatorname{Re}[(g(z)-g(v))(\bar{z}-\bar{v})] \geq m|z-v|^{2}$,

(ii) $\operatorname{Im}(g(z) \bar{z})=0$,

(iii) $|g(z)| \leq M|z|$

for all $v, z \in \mathbb{C}$ and for some constants $m, M \in \mathbb{R}_{+}$.

Examples of functions satisfying Assumption 2.1 can be found in the literature for wave and Schrödinger equations (see for example [42]). In particular, assumptions (i) and (iii) form a complex analog to the assumption of monotonicity that appears in the study of wave equations.

When we consider the model in (2.10), we will assume $f: V \rightarrow V$ to be Lipschitz continuous in the sense that there exists a constant $L$ such that for every pair $u, v \in V$,

$$
\|f(u)-f(v)\|_{V} \leq L\|u-v\|_{V} .
$$

Now, the well-posedness of (2.10) is achieved by considering a more general Wentzell ibvp. Namely, we replace $g\left(u_{t}\right)$ on the boundary with $g((\lambda+i \alpha) \Delta u+h(u)+\gamma u)$, where we assume $h: H^{1}(\Omega) \rightarrow L^{2}\left(\Gamma_{1}\right)$ is Lipschitz in the sense

$$
\|h(u)-h(v)\|_{H^{1 / 2}\left(\Gamma_{1}\right)} \leq K\|u-v\|_{V}
$$

for some $K>0$. 
Since the trace operator $\gamma_{0}: H^{1}(\Omega) \rightarrow L^{2}(\Gamma)$ is continuous and linear, this formulation actually generalizes problem (2.10), which is the special case where $h(u)=\gamma_{0}(f(y))$. In order to recast the problem in an abstract operator theoretic form, we define the operator $A_{f}$ given by

$$
A_{f} u=(\lambda+i \alpha) \Delta u+f(u)
$$

with the domain

$$
D\left(A_{f}\right)=\left\{y \in V, \Delta y \in V, \frac{\partial y}{\partial \nu}=-g\left(\left.(\lambda+i \alpha) \Delta\right|_{\Gamma_{1}} y+h(y)\right) \text { on } \Gamma_{1}\right\}
$$

where $g$ satisfies Assumption 2.1, and $f$ and $h$ satisfy (2.11) and (2.12), respectively. One should notice that if $f$ satisfies (2.11), then $h \equiv \gamma_{0} f$ satisfies (2.12) by the Sobolev trace inequality.

The following well-posedness fact holds true.

Theorem 2.4 (Nonlinear Perturbations I). Taking into account Assumption 2.1, (2.11) and (2.12), the operator $\left(A_{f}, D\left(A_{f}\right)\right)$ generates a strongly continuous semigroup on $V$.

Going back to the idbvp (2.10), one obtains:

Corollary 2.2 (Nonlinear Perturbations II). Under the same assumptions in Theorem 2.4, for any initial data $u_{0} \in V$ there exists a unique solution $u \in C([0, \infty), V)$ of the problem $(2.10)$.

Finally, we study the problem (1.1) with $g \equiv i d$, that is,

$$
\begin{cases}u_{t}-(\lambda+i \alpha) \Delta u+(\kappa+i \beta)|u|^{p-1} u-\gamma u=0 & \text { in } \Omega \times \mathbb{R}_{+}, \\ \frac{\partial u}{\partial \nu}=-u_{t} & \text { on } \Gamma_{1} \times \mathbb{R}_{+}, \\ u=0 & \text { on } \Gamma_{0} \times \mathbb{R}_{+}, \\ u(0)=u_{0} & \text { in } \Omega .\end{cases}
$$

In order to achieve our goal, we resort to the inhomogeneous linear theory with a forcing term given by $F(u)=-(\kappa+i \beta)|u|^{p-1} u+\gamma u$. Using a contraction mapping argument and some a priori estimates, we prove the following local well-posedness result:

Theorem 2.5 (Local Strong Solutions). Let $N \leq 3$ and $\beta>0$. Then, for every bounded subset $B \subset X_{0}$, there exists $T>0$ such that for all $\left(u_{0}, w_{0}\right) \in B$, there exists a unique solution $u$ of $(2.15)$ with time derivative $u_{t}=w$ such that the pair $(u, w) \in X_{T}$.

Spaces $X_{0}$ and $X_{T}$ are defined in Section 6. Given the association $w=u_{t}$, we can rephrase $(u, w) \in X_{T}$ as

$$
u \in C\left([0, T] ; H^{2}(\Omega) \cap V\right) \cap C^{1}([0, T] ; V) .
$$

Regarding the global well-posedness, we have the following result:

Theorem 2.6 (Global Strong Solutions). Let $\left(u, u_{t}\right) \in X_{T}$ be a local strong solution as in Theorem 2.5 and $\beta>0$. Then, this solution can be extended globally under the conditions: $p \geq 2$ if $N=1 ; p \in[2,5]$ if $N=2$; and $p \in\left[2, \frac{11}{3}\right]$ if $N=3$. 
Relaxing the smoothness assumption on $u_{0}$ a little bit, one can get solutions continuous in time at the $H^{1}$-level. We first define the notion of weak solutions as follows.

Definition 2.1 (Notion of Weak Solutions). We say that $u$ is a weak solution of problem (2.15) if given $T>0$ and

$$
u_{0} \in Q \equiv\left\{\varphi \in V \text { such that } \gamma_{0} \varphi \in L^{p+1}\left(\Gamma_{1}\right)\right\}
$$

$\left.u\right|_{t=0}=u_{0}$ and there exists a sequence of global strong solutions $u_{\mu}$ with initial data $u_{\mu, 0}$ such that $u_{\mu, 0} \rightarrow u_{0}$ in $Q$ and $u_{\mu} \rightarrow u$ in $C([0, T], V) \cap L^{2}\left(0, T ; H^{2}(\Omega)\right), u_{\mu}^{\prime} \rightarrow u^{\prime}$ in $L^{2}\left(0, T ; L^{2}(\Omega)\right)$, and $\gamma_{0} u_{\mu}^{\prime} \rightarrow \gamma_{0} u^{\prime}$ in $L^{2}\left(0, T ; L^{2}\left(\Gamma_{1}\right)\right)$.

Our next result is the following theorem on the existence and uniqueness of weak solutions:

Theorem 2.7 (Weak Solutions). Let $N \leq 3, \beta>0, u_{0} \in V$ so that $\gamma_{0} u_{0} \in L^{p+1}\left(\Gamma_{1}\right)$ and $(p, N)$ satisfy the conditions given in Theorem 2.6. Then, problem (2.15) possesses a unique (weak) solution in the sense of Definition 2.1.

Remark 2.2. Theorem 2.7 gives a more regular weak solution than that obtained in the corresponding Schrödinger problem in [34], where the weak solution only satisfies $u \in L^{\infty}(0, T ; V)$ with $u^{\prime} \in L^{2}\left(0, T ; V^{\prime}\right)$. Moreover, the uniqueness in NLS is proved only in the case that the nonlinearity is globally Lipschitz in $V$, whereas for the CGLE, uniqueness is proved in a more general setting, thanks to the smoothing effect of the parabolic component of the Ginzburg-Landau operator.

At this point, a natural question is to ask whether the solutions of the CGLE with dynamic boundary conditions get close to the solutions of NLS with same type of boundary conditions as the parameter pair $\epsilon \equiv(\lambda, \kappa) \rightarrow 0$. We show that this is indeed true by the following theorems.

Theorem 2.8 (Inviscid Limits I). Suppose that $u_{\epsilon}$, where $\epsilon=(\lambda, \kappa)$, is a global (weak) solution to the idbvp for the CGLE with the initial condition $u_{0} \in Q$ as in Theorem 2.7. Then, there exists $u \in L^{\infty}(0, T ; V)$ with $u_{t} \in L^{2}\left(0, T ; V^{\prime}\right)$ such that a subsequence of $u_{\epsilon}$ (still denoted same) satisfies

$$
\begin{aligned}
u_{\epsilon} & \rightarrow u \text { weakly star in } L^{\infty}(0, T ; V), \\
\partial_{t} u_{\epsilon} & \rightarrow u_{t} \text { weakly in } L^{2}\left(0, T ; V^{\prime}\right)
\end{aligned}
$$

as $\epsilon \rightarrow 0$, and most importantly u solves the idbvp for the NLS in the weak sense.

Theorem 2.9 (Inviscid Limits II). Let $N=2$ and $p=3$. Suppose that $u_{\epsilon}$ is a global strong solution of the idbvp for the CGLE with the initial condition $u_{\epsilon}^{0}$ and $u$ is a global strong solution of the idbvp for the NLS with initial condition $u_{0}$ such that $u_{\epsilon}^{0} \rightarrow u_{0}$ in $V$ as $\epsilon=(\lambda, \kappa) \rightarrow 0$. Then,

$$
\left\|u_{\epsilon}-u\right\|_{V}=O(\lambda)+O(\kappa)
$$

as $\epsilon=(\lambda, \kappa) \rightarrow 0$.

Finally, we prove that the solutions of the idbvp for the CGLE decay to zero exponentially fast if $\gamma \leq 0$. This is easy to prove with $\gamma<0$, and we have the following theorem. 
Theorem 2.10 (Stabilization I). Let

$$
u_{0} \in Q \equiv\left\{\varphi \in V \cap L^{p+1}(\Omega) \text { such that } \gamma_{0} \varphi \in L^{p+1}\left(\Gamma_{1}\right)\right\}
$$

and $u$ be the corresponding global weak solution of the idbvp for the CGLE (Eq. (2.15)) with $\gamma<0$ as in Theorem 2.7. Then,

$$
F(t) \leq E_{0} e^{-|\gamma| t} \text { for } t \geq 0
$$

where

$$
F(t) \equiv \frac{\alpha}{2}\|\nabla u(t)\|_{L^{2}(\Omega)}^{2}+\frac{\beta}{p+1}\|u(t)\|_{L^{p+1}(\Omega)}^{p+1}
$$

and

$$
\begin{aligned}
E_{0} \equiv & \frac{\alpha}{2}\left\|\nabla u_{0}\right\|_{L^{2}(\Omega)}^{2}+\frac{\beta}{p+1}\left\|u_{0}\right\|_{L^{p+1}(\Omega)}^{p+1}-\frac{\alpha \gamma}{2}\left\|u_{0}\right\|_{L^{2}\left(\Gamma_{1}\right)}^{2} \\
& +\frac{1}{p+1}(\alpha \kappa+\beta \lambda)\left\|u_{0}\right\|_{L^{p+1}\left(\Gamma_{1}\right)}^{p+1} .
\end{aligned}
$$

Remark 2.3. The problem $\gamma=0$ is more challenging and requires control theoretic tools. In the case $\gamma=0$, there is usually no decay for the complex Ginzburg-Landau equation even at $L^{2}$-level. However, in our model the dynamic boundary input plays the role of a stabilizing control/feedback and one actually gains an exponential decay of solutions. In fact, we have the following theorem.

Theorem 2.11 (Stabilization II). Let

$$
u_{0} \in Q \equiv\left\{\varphi \in V \cap L^{p+1}(\Omega) \text { such that } \gamma_{0} \varphi \in L^{p+1}\left(\Gamma_{1}\right)\right\}
$$

and $u$ be the corresponding global weak solution of the idbvp for the CGLE (Eq. (2.15)) with $\gamma=0$ as in Theorem 2.7. Moreover, suppose that $\Omega$ satisfies the following geometric condition: $\exists x_{0} \in \mathbb{R}^{N}$ such that $\left(x-x_{0}\right) \cdot \nu \leq 0$ on $\Gamma_{0}$ and $\left(x-x_{0}\right) \cdot \nu>0$ on $\Gamma_{1}$. Then, there exists some $C>0$ such that

$$
F(t) \leq F(0) e^{1-\frac{t}{C}} \text { for } t \geq 0
$$

where $F(t)$ is given in (2.19).

\section{Linear homogeneous problem}

In this section, our aim is to prove Theorems 2.1 and 2.2. The proof of these results is based on a method developed in [34] adapted for the idbvp (2.6). In order to achieve this, we convert the idbvp (2.6) into the Wentzell ibvp in (2.7). This is accomplished by a suitable use of semigroup proving that the operator $A$ is maximal dissipative.

\section{Dissipativity:}

Proof of the dissipativity is standard with exception of the computations that shows the presence of the boundary condition on $\Gamma_{1}$. First of all, we observe that the operator $A$ is not dissipative on space $L^{2}(\Omega)$. Indeed, from the definition of the operator $A$ given in (2.4), taking into account that $\frac{\partial u}{\partial \nu}=-(\lambda+i \alpha) \Delta u$ on $\Gamma_{1}$ (see (2.7)), we have

$$
\operatorname{Re}(A u, u)_{L^{2}(\Omega)}=-\lambda\|\nabla u\|_{L^{2}(\Omega)}^{2}-\operatorname{Re}\left[(\lambda+i \alpha)^{2}(\Delta u, u)_{L^{2}\left(\Gamma_{1}\right)}\right], \forall u \in D(A),
$$

which it is not clear from the above inequality that $\operatorname{Re}(A u, u)_{L^{2}(\Omega)} \leq 0$. 
So, performing the scalar product of the space $V$ instead $L^{2}(\Omega)$, for any $u \in D(A)$, we obtain

$$
\begin{aligned}
(A u, u)_{V} & =(\nabla A u, \nabla u)_{L^{2}(\Omega)} \\
& =(\lambda+i \alpha)(\nabla \Delta u, \nabla u)_{L^{2}(\Omega)} \\
& =-(\lambda+i \alpha)(\Delta u, \Delta u)_{L^{2}(\Omega)}+(\lambda+i \alpha)\left(\Delta u, \frac{\partial u}{\partial \nu}\right)_{L^{2}\left(\Gamma_{1}\right)} \\
& =-(\lambda+i \alpha)\|\Delta u\|_{L^{2}(\Omega)}^{2}-\left\|\frac{\partial u}{\partial \nu}\right\|_{L^{2}\left(\Gamma_{1}\right)}^{2},
\end{aligned}
$$

where in the last equality we used the fact that $\frac{\partial u}{\partial \nu}=-(\lambda+i \alpha) \Delta u$ on the boundary $\Gamma_{1}$. Taking the real parts in (3.2), we obtain the desired, that is,

$$
\operatorname{Re}(A u, u)_{V}=-\lambda\|\Delta u\|_{L^{2}(\Omega)}^{2}-\left\|\frac{\partial u}{\partial \nu}\right\|_{L^{2}\left(\Gamma_{1}\right)}^{2} \leq 0 .
$$

\section{Maximality:}

For this purpose, as well as proceeded in [34]. We define the following Banach space (see [34, Lemma 2.1]):

$$
Z=\left\{z \in V, \Delta z \in L^{2}(\Omega), \frac{\partial z}{\partial \nu} \in L^{2}\left(\Gamma_{1}\right)\right\}
$$

which is equipped with the norm

$$
\|z\|_{Z}^{2}=\|z\|_{V}^{2}+\|\Delta z\|_{L^{2}(\Omega)}^{2}+\left\|\frac{\partial z}{\partial \nu}\right\|_{L^{2}\left(\Gamma_{1}\right)}^{2} .
$$

Now, recalling the definition of $A$ and integrating by parts, let us define the following bilinear form

$$
a(u, z) \equiv(\lambda+i \alpha)(\Delta u, \Delta z)_{L^{2}(\Omega)}+\left(\frac{\partial u}{\partial \nu}, \frac{\partial z}{\partial \nu}\right)_{L^{2}\left(\Gamma_{1}\right)}+\theta(\nabla u, \nabla z)_{L^{2}(\Omega)} .
$$

We desire guarantee that the identity

$$
a(u, z)=(-A u+\theta u, z)_{V}
$$

will hold true whenever $u \in D(A)$ and $z \in V$, where $\theta$ will be chosen later. To this end, we employ the Browder-Minty theorem [43, Theorem 5.16] to show that for any fixed $f \in V$, there exists a unique (weak) solution $u \in V$ satisfying the following variational form

$$
a(u, z)=(-f, z)_{V}, \forall z \in V .
$$

The next step is to prove that $a(u, z)$ is continuous and coercive on $Z$. We are able to prove that

$$
\begin{aligned}
|a(u, z)| & \leq C(\alpha, \lambda, \theta)\|u\|_{Z}\|z\|_{Z}, \\
|a(u, u)|^{2} & \geq C(\alpha, \gamma, \lambda, \theta)\|u\|_{Z}^{2}, \quad \text { considering } \operatorname{Re}(\theta)>0 .
\end{aligned}
$$

These computations can be done as in [34], therefore omitted here.

So, Browder-Minty theorem says us that for all $f \in Z^{\prime}$, there exists a solution $v \in Z$ satisfying (3.8). Moreover, from the chain $D(A) \subset Z \subset V \subset Z^{\prime}$, we conclude that for all $f \in V$ there is a solution $v \in Z \subset V$.

Beyond that, by testing the variational form with $z \in Z$ satisfying $\frac{\partial z}{\partial \nu}=0$, it follows that

$$
(\lambda+i \alpha)(\Delta v, \Delta z)_{L^{2}(\Omega)}-\theta(v, \Delta z)_{L^{2}(\Omega)}=(f, \Delta z)_{L^{2}(\Omega)},
$$


then,

$$
(\lambda+i \alpha) \Delta v-\theta v=f
$$

Since $v, f \in V$, it follows that $\Delta v \in V$. Using the variational form once more, one recovers the boundary condition so that $v \in D(A)$. This tells us that the operator $A$ is the infinitesimal generator of a $C_{0}$ semigroup of contractions on $V$.

The fact that $\Delta v \in V$ implies that $\left.\Delta v\right|_{\Gamma_{1}} \in H^{1 / 2}\left(\Gamma_{1}\right)$, that is, $\frac{\partial v}{\partial \nu} \in H^{1 / 2}\left(\Gamma_{1}\right)$. Trace theory says us that $v \in H^{2}(\Omega)$ which we can conclude that the regularity of $D(A)$ is at least at the level of $H^{2}(\Omega)$. Since $D(A)$ is dense in $V$, from the Lumer-Philips theorem or [44, Corollary IV.3.2,], Theorem 2.1 follows. Translating the Wentzell ibvp (2.7) back into the idbvp (2.6), the proof Theorem 2.2 is completed.

\section{Linear nonhomogeneous problem}

In this section, our aim is to prove Theorem 2.3 and Corollary 2.1. Again, we borrowed the ideas used in [34]. Now, we consider the problem (2.8). First of all, according to [44, Theorem IV.4.1A], we can deduce the following result:

Proposition 4.1. If $f \in L^{1}(0, \infty ; V)$ and $u_{0} \in \overline{D(A)}=V$, there exists a unique generalized solution $u \in C([0, \infty) ; V)$ to the problem $(2.8)$.

Now, we want to consider the general Wentzell ibvp in (2.9). Let us first consider the case $f=0$ and then employ the superposition principle to obtain the well-posedness of (2.9). First, we define the Neumann map $\mathcal{N}$ below where for a given $g \in H^{s}\left(\Gamma_{1}\right), \mathcal{N} g$ solves

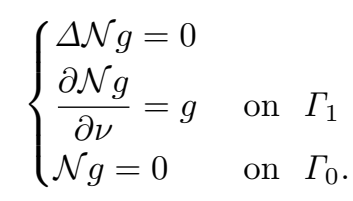

Using the elliptic theory [45], one can deduce

$$
\mathcal{N}: H^{s}\left(\Gamma_{1}\right) \rightarrow H^{s+3 / 2}(\Omega) \quad \text { is continuous for all } s \in \mathbb{R} .
$$

From above, define $\tilde{u}=u-\mathcal{N} g$. Making use of the boundary condition on $\Gamma_{1}$ given in (2.9) and the conditions $\Delta \mathcal{N} g=0$ and $\frac{\partial \mathcal{N} g}{\partial \nu}=g$ on $\Gamma_{1}$, it follows that

$$
\begin{aligned}
\frac{\partial \tilde{u}}{\partial \nu} & =\frac{\partial u}{\partial \nu}-\frac{\partial \mathcal{N} g}{\partial \nu} \\
& =-(\lambda+i \alpha) \Delta u+(\lambda+i \alpha) \underbrace{\Delta \mathcal{N} g}_{=0} \\
& =-(\lambda+i \alpha) \Delta \tilde{u} \quad \text { on } \Gamma_{1} .
\end{aligned}
$$

Moreover, since we are considering the case $f=0$, we see that

$$
\tilde{u}_{t}=(\lambda+i \alpha) \Delta u-\mathcal{N} g_{t}
$$

and making use of the fact that $\Delta \mathcal{N} g=0$, we have

$$
\tilde{u}_{t}=(\lambda+i \alpha) \Delta \tilde{u}-\mathcal{N} g_{t} .
$$


Combining (4.3) and (4.4), the problem with respect to the function $\tilde{u}$ becomes:

$$
\begin{cases}\tilde{u}_{t}=(\lambda+i \alpha) \Delta \tilde{u}-\mathcal{N} g_{t} & \text { in } \Omega \times(0, \infty) \\ \tilde{u}=0 & \text { on } \Gamma_{0} \times(0, \infty) \\ \frac{\partial \tilde{u}}{\partial \nu}=-(\lambda+i \alpha) \Delta \tilde{u} & \text { on } \Gamma_{1} \times(0, \infty) \\ \tilde{u}(0)=u_{0}-\mathcal{N} g(0) . & \end{cases}
$$

The above problem gives us the following result whose proof can be done as in [[34], Lemma 2.3]:

Lemma 4.1. If $g \in W^{1,1}\left(0, \infty ; H^{-1 / 2}\left(\Gamma_{1}\right)\right)$, then there exists a unique solution $u \in C([0, \infty) ; V)$ to the problem (2.9).

Below we will prove Theorem 2.3. This result requires only $g \in L^{2}\left(0, T ; L^{2}\left(\Gamma_{1}\right)\right)$. Indeed, Lemma 4.1 tells us that there exists a unique solution $u \in C([0, T] ; V)$ to the problem (2.9) whenever $g \in$ $W^{1,1}\left(0, T ; H^{-1 / 2}\left(\Gamma_{1}\right)\right)$. So it is sufficient to prove that $\sup _{t \in[0, T]}\|u(t)\|_{V}<\infty$ for $g \in L^{2}\left(0, T ; L^{2}\left(\Gamma_{1}\right)\right)$ and then use a density argument.

Multiplying (2.9) by $\bar{u}$ in $V$, taking the inner product, and integrating in temporal variable $t$, it follows that

$$
\int_{0}^{t}\left(u_{t}(s), u(s)\right)_{V} d s-\int_{0}^{t}((\lambda+i \alpha) \Delta u(s), u(s))_{V} d s=\int_{0}^{t}(f(s), u)_{V} d s .
$$

Without loss of generality, we take $f=0$. The general case $f \neq 0$ can be solved a posteriori via superposition. We observe that the first term satisfies

$$
\operatorname{Re} \int_{0}^{t}\left(u_{t}(s), u(s)\right)_{V} d s=\frac{1}{2} \int_{0}^{t} \frac{d}{d t}\|u(s)\|_{V}^{2} d s=\frac{1}{2}\|u(t)\|_{V}^{2}-\frac{1}{2}\|u(0)\|_{V}^{2} .
$$

Using integration by parts on the second term at the left hand side of (4.6) we get

$$
\begin{aligned}
& \int_{0}^{t}((\lambda+i \alpha) \Delta u(s), u(s))_{V} d s \\
& =\int_{0}^{t}\left[-(\lambda+i \alpha)\|\Delta u(s)\|_{L^{2}(\Omega)}^{2}+(\lambda+i \alpha)\left(\Delta u(s), \frac{\partial u}{\partial \nu}(s)\right)_{L^{2}\left(\Gamma_{1}\right)}\right] d s .
\end{aligned}
$$

Now, substituting the boundary condition on $\Gamma_{1}$, namely, $(\lambda+i \alpha) \Delta u=g-\frac{\partial u}{\partial \nu}$ we obtain

$$
\begin{aligned}
& \int_{0}^{t}\left((\lambda+i \alpha) \Delta u(s), \frac{\partial u}{\partial \nu}(s)\right)_{L^{2}\left(\Gamma_{1}\right)} d s \\
& =\int_{0}^{t}\left[-\left\|\frac{\partial u}{\partial \nu}(s)\right\|_{L^{2}\left(\Gamma_{1}\right)}^{2}+\left(g, \frac{\partial u}{\partial \nu}(s)\right)_{L^{2}\left(\Gamma_{1}\right)}\right] d s .
\end{aligned}
$$

Substituting (4.7)-(4.9) in (4.6) and taking the real parts, we get:

$$
\begin{aligned}
0 & =\frac{1}{2}\|u(t)\|_{V}^{2}-\frac{1}{2}\|u(0)\|_{V}^{2}+\lambda \int_{0}^{t}\|\Delta u(s)\|_{L^{2}(\Omega)}^{2} d s+\int_{0}^{t}\left\|\frac{\partial u}{\partial \nu}(s)\right\|_{L^{2}\left(\Gamma_{1}\right)}^{2} d s \\
& -\operatorname{Re} \int_{0}^{t}\left(g, \frac{\partial u}{\partial \nu}(s)\right)_{L^{2}\left(\Gamma_{1}\right)} d s .
\end{aligned}
$$

We infer that

$$
\begin{aligned}
& \frac{1}{2}\|u(t)\|_{V}^{2}+\lambda \int_{0}^{t}\|\Delta u(s)\|_{L^{2}(\Omega)}^{2} d s+\left(1-\frac{1}{4 \eta}\right) \int_{0}^{t}\left\|\frac{\partial u}{\partial \nu}(s)\right\|_{L^{2}\left(\Gamma_{1}\right)}^{2} d s \\
& \leq \frac{1}{2}\|u(0)\|_{V}^{2}+\eta \int_{0}^{t}\|g\|_{L^{2}\left(\Gamma_{1}\right)}^{2} d s
\end{aligned}
$$

where we choose $\eta>\frac{1}{4}$. 
More generally, for $f \in L^{1}(0, T ; V)$, we observe

$$
\begin{aligned}
& \frac{1}{4}\|u(t)\|_{V}^{2}+\lambda \int_{0}^{t}\|\Delta u(s)\|_{L^{2}(\Omega)}^{2} d s+\left(1-\frac{1}{4 \eta}\right) \int_{0}^{t}\left\|\frac{\partial u}{\partial \nu}(s)\right\|_{L^{2}\left(\Gamma_{1}\right)}^{2} d s \\
& \leq \frac{1}{2}\|u(0)\|_{V}^{2}+\eta \int_{0}^{t}\|g\|_{L^{2}\left(\Gamma_{1}\right)}^{2} d s+\int_{0}^{t}\|f\|_{V}^{2} d s \\
& \leq C\left(u_{0}, f, g\right) .
\end{aligned}
$$

By making the identification $g=\left.f\right|_{\Gamma_{1}}$, we can identify (2.9) with the idbvp (2.8). Note that if $f \in L^{2}(0, T ; V)$, by trace theory $g \in L^{2}\left(0, T ; H^{1 / 2}\left(\Gamma_{1}\right)\right)$. Moreover, since $\Delta u \in L^{2}\left(0, T ; L^{2}(\Omega)\right)$ and $f \in L^{2}(0, T ; V)$, we have that $u_{t} \in L^{2}\left(0, T ; L^{2}(\Omega)\right)$, and this concludes the proof of Corollary 2.1.

\section{Remark 4.1.}

(i) Given $u \in H^{1}(\Omega)$, recalling the trace theory, one gets that $\frac{\partial u}{\partial \nu} \in H^{-1 / 2}(\Gamma)$. Therefore, the additional regularity $\frac{\partial u}{\partial \nu} \in L^{2}\left(\Gamma_{1}\right)$, which shows up in Theorem 2.3, is a "hidden" regularity due to the underlying Wentzell structure.

(ii) Again, just by a formal argument, one would expect $\Delta u \in H^{-1}(\Omega)$. The fact that $\Delta u \in L^{2}(\Omega)$, is a result of the smoothing effect due to the intrinsic properties of the parabolic component of the Ginzburg-Landau operator.

(iii) From Corollary 2.1, we also conclude that the map

$$
\left(f, u_{0}\right) \longmapsto u
$$

is bounded from $L^{2}(0, \infty, V) \times V$ into $C([0, \infty) ; V)$.

Next, we desire to obtain a Duhamel's formula for a problem derived from (2.9) and an estimate for $u_{t}$. The next two results, namely, Lemma 4.2 and Theorem 4.1 can be obtained employing similar ideas used in [[34], Lemma 2.7 and Theorem 2.8], therefore their respective proofs will be omitted here.

Lemma 4.2. Let $u_{0}, f$ and $g$ satisfy

$$
\begin{array}{ll}
\text { (i) } u_{0} \in V & \text { (ii) } f \in L^{1}(0, T ; V) \\
\text { (iii) } g \in L^{2}\left(0, T ; L^{2}\left(\Gamma_{1}\right)\right) . &
\end{array}
$$

Then, the solution $u \in C([0, T] ; V)$ of $(2.9)$ can be represented by

$$
u(t)=e^{A t} u_{0}-A \int_{0}^{t} e^{A(t-s)} \mathcal{N} g(s) d s+\int_{0}^{t} e^{A(t-s)} f(s) d s .
$$

Remark 4.2. Combining Lemma 4.2 and Theorem 2.3, we deduce that the following map is continuous:

$$
\begin{aligned}
\mathcal{L}: L^{2}\left(0, T ; L^{2}\left(\Gamma_{1}\right)\right) & \rightarrow C([0, T] ; V) \\
g & \mapsto A \int_{0}^{t} e^{A(t-s)} \mathcal{N} g(s) d s .
\end{aligned}
$$

Below, we will be looking at more regular solutions corresponding to the inhomogeneous problem (2.9). We start our discussion with the following result:

Theorem 4.1. In addition to the assumptions in Lemma 4.2 assume also that:
(i) $f_{t} \in L^{1}(0, T ; V)$
(iii) $\Delta u_{0} \in V$ and $\frac{\partial u_{0}}{\partial \nu}-g(0)=-(\lambda+i \alpha) \Delta u_{0}$
(ii) $g_{t} \in L^{2}\left(0, T ; L^{2}\left(\Gamma_{1}\right)\right)$. 
Then, the following estimate is satisfied:

$$
\left\|u_{t}\right\|_{C([0, T] ; V)} \leq C\left[\|f\|_{W^{1,1}(0, T ; V)}+\|g\|_{H^{1}\left(0, T ; L^{2}\left(\Gamma_{1}\right)\right)}+\left\|\Delta u_{0}\right\|_{V}+\left\|u_{0}\right\|_{V}\right] .
$$

If, in addition, $g \in C\left([0, T] ; H^{1 / 2}\left(\Gamma_{1}\right)\right)$, then $u \in C\left([0, T] ; H^{2}(\Omega)\right)$.

Remark 4.3. The proof of Theorem 4.1 brings us useful informations (for more details of these facts, see [34]) that will be employed later, namely:

(i) We notice that the conditions imposed on the initial data are equivalent to saying that $u_{0}-\mathcal{N} g(0) \in$ $D(A)$. Indeed, this follows from noticing that $u_{0}-\mathcal{N} g(0) \in D(A)$ translates into the following conditions:

$$
\begin{cases}\frac{\partial\left(u_{0}-\mathcal{N} g(0)\right)}{\partial \nu} & =-(\lambda+i \alpha) \Delta\left(u_{0}-\mathcal{N} g(0)\right) \\ \Rightarrow \frac{\partial u_{0}}{\partial \nu}-g(0) & =-(\lambda+i \alpha) \Delta u_{0} \\ \Delta\left(u_{0}-\mathcal{N} g(0)\right) & \equiv \Delta u_{0} \in V\end{cases}
$$

So, thanks to the compatibility condition described in (4.17), and due to [46, Theorem 2.4(c)], we have

$$
A e^{A t}\left[u_{0}-\mathcal{N} g(0)\right]=e^{A t} A\left[u_{0}-\mathcal{N} g(0)\right]=(\lambda+i \alpha) e^{A t} \Delta u_{0} .
$$

(ii) Making use of the semigroup formula given in Lemma 4.2, we infer:

$$
u_{t}(t)=A e^{A t}\left[u_{0}-\mathcal{N} g(0)\right]+e^{A t} f(0)+\int_{0}^{t} e^{A(t-s)} f_{t}(s) d s-\mathcal{L} g_{t}(t),
$$

where $\mathcal{L}$ is the map given in (4.15).

Remark 4.4. The function $u$ obtained above in the class $C\left([0, \infty) ; H^{2}(\Omega)\right)$ is a solution to the problem (2.9), that is,

$$
\begin{cases}(\lambda+i \alpha) \Delta u=u_{t}+f & \text { in } \Omega \times[0, T] \\ \frac{\partial u}{\partial \nu}=-(\lambda+i \alpha) \Delta u+g & \text { on } \Gamma_{1} \times[0, T]\end{cases}
$$

so that $f \in V \hookrightarrow L^{2}(\Omega)$ and $g \in H^{1 / 2}\left(\Gamma_{1}\right)$. So, denoting $C=C(\alpha, \lambda, T)$, from the continuity of the trace map $\gamma_{0}: H^{1}(\Omega) \rightarrow H^{1 / 2}\left(\Gamma_{1}\right)$ and (4.20), we obtain:

$$
\begin{aligned}
\|u(t)\|_{H^{2}(\Omega)} & \leq C\left(\left\|u_{t}\right\|_{L^{2}(\Omega)}+\|f\|_{L^{2}(\Omega)}+\|g\|_{H^{1 / 2}\left(\Gamma_{1}\right)}+|\lambda+i \alpha|\|\Delta u\|_{H^{1 / 2}\left(\Gamma_{1}\right)}\right) \\
& \leq C\left(\left\|u_{t}\right\|_{V}+\|f\|_{V}+\|g\|_{H^{1 / 2}\left(\Gamma_{1}\right)}+\|\Delta u\|_{V}\right) \\
& \leq C\left(\left\|u_{t}\right\|_{V}+\|f\|_{V}+\|g\|_{H^{1 / 2}\left(\Gamma_{1}\right)}\right)
\end{aligned}
$$

Combining Lemma 4.2, (4.16) and (4.21), we get

$$
\begin{aligned}
\left\|u_{t}\right\|_{C([0, T] ; V)}+\|u\|_{C\left([0, T] ; H^{2}(\Omega)\right)} & \leq C\left[\|f\|_{H^{1}(0, T ; V)}+\|g\|_{H^{1}\left(0, T ; H^{1 / 2}\left(\Gamma_{1}\right)\right)}\right. \\
& \left.+\left\|\Delta u_{0}\right\|_{V}+\left\|u_{0}\right\|_{V}\right] .
\end{aligned}
$$

The above estimate applied to idbvp yields the following:

Theorem 4.2. With reference to (2.8), in addition to the assumptions in Corollary 2.1 assume also that: 
(i) $f \in H^{1}(0, T ; V)$

(ii) $\Delta u_{0} \in V$ and $\frac{\partial u_{0}}{\partial \nu}-\left.f\right|_{\Gamma_{1}}(0)=-(\lambda+\alpha i) \Delta u_{0}$.

Then $u_{t} \in C([0, T], V)$ with appropriate control of the estimates, that is,

$$
\left\|u_{t}\right\|_{C([0, T] ; V)}+\|u\|_{C\left([0, T] ; H^{2}(\Omega)\right)} \leq C\left[\|f\|_{H^{1}(0, T ; V)}+\left\|\Delta u_{0}\right\|_{V}+\left\|u_{0}\right\|_{V}\right] .
$$

Proof. The proof can be done as in Theorem 2.9 in [34], therefore, omitted here.

\section{Nonlinear perturbations}

Our aim in this section is to prove Theorem 2.4 and Corollary 2.2. We will construct solutions for the nonlinear model (2.10), where $g(z)$ satisfies the conditions given in Assumption 2.1. Moreover, the function $f$ is assumed to satisfy (2.11). As in previous sections, the well-posedness is obtained by converting this idbvp into a Wentzell ibvp. Namely, we replace $g\left(u_{t}\right)$ on the boundary with $g((\lambda+i \alpha) \Delta u+h(u))$, where the function $h$ satisfies (2.12). Here, we consider the operator $A_{f}$ given in (2.13) with its domain characterized by (2.14). The proof of theorem (2.14) is based on an extended monotonicity method developed in the linear case. From the outset, we note that this proof is strongly patterned along Section 3 in [34] and therefore, we present the details necessary regarding the ibvp.

From above, we are able to prove the $\omega$-maximal dissipativity of the operator $A_{f}$ :

Dissipativity: From definition of the operator $A_{f}$ given in (2.13), we infer

$$
\begin{aligned}
\operatorname{Re}\left(A_{f} u-A_{f} v, u-v\right)_{V} & \leq-\lambda\|\Delta u-\Delta v\|_{L^{2}(\Omega)}^{2}+\left(\frac{1}{4 \eta}-m\right)\left\|\frac{\partial u}{\partial \nu}-\frac{\partial v}{\partial \nu}\right\|_{L^{2}\left(\Gamma_{1}\right)}^{2} \\
& +\left[\eta K^{2}+L\right]\|u-v\|_{V}^{2} .
\end{aligned}
$$

Now, since $\lambda>0$, by taking $\omega>\eta K^{2}+L$ with $\eta$ large enough, we may conclude that

$$
\operatorname{Re}\left(A_{f} u-A_{f} v-\omega I(u-v), u-v\right)_{V} \leq 0,
$$

which proves that the operator $A$ is $\omega$-dissipative.

\section{Maximality:}

At this point, we consider the space $Z$ given in (3.4). Now, we define

$$
\begin{aligned}
a(u, v)=\theta(u, v)_{V} & +(\lambda+i \alpha)(\Delta u, \Delta v)_{L^{2}(\Omega)}+\left(g^{-1}\left(\frac{\partial u}{\partial \nu}\right), \frac{\partial v}{\partial \nu}\right)_{L^{2}\left(\Gamma_{1}\right)} \\
& -(f(u), v)_{V}+\left(h(u), \frac{\partial v}{\partial \nu}\right)_{L^{2}\left(\Gamma_{1}\right)}
\end{aligned}
$$

We borrow the procedure employed in [34] to show that this form is continuous and coercive so that the Browder-Minty theorem can be applied. This will imply that, for every $j \in V \subset Z^{\prime}$, there exists a unique $u \in Z$ satisfying

$$
a(u, v)=(-j, v)_{V} \text { for all } v \in Z,
$$

for some value of $\theta$ such that $\operatorname{Re}(\theta)$ is sufficiently large.

Regarding the continuity, it can be proved that there exists a bound $C(\lambda, \theta, \alpha, M, L, K)$ such that

$$
|a(u, v)| \leq C(\lambda, \theta, \alpha, M, L, K)\|u\|_{Z}\|v\|_{Z} .
$$


For coercivity, we get the following estimate:

$$
\begin{aligned}
|a(u, u)| & \geq \frac{\sqrt{2}}{2} \operatorname{Re}(\theta)\|u\|_{V}^{2}+\frac{\sqrt{2}}{2}(\lambda+\alpha)\|\Delta u\|_{L^{2}(\Omega)}^{2}+\left(\frac{\sqrt{2} m}{2}-\frac{1}{4 \eta}\right)\left\|\frac{\partial u}{\partial \nu}\right\|_{L^{2}\left(\Gamma_{1}\right)}^{2} \\
& -\sqrt{2}\left[L+\eta K^{2}\right]\|u\|_{V}^{2} \\
& =\frac{\sqrt{2}}{2}\left[\operatorname{Re}(\theta)-2 L-2 \eta K^{2}\right]\|u\|_{V}^{2}+(\lambda+\alpha)\|\Delta u\|_{L^{2}(\Omega)}^{2}+\left(\frac{\sqrt{2} m}{2}-\frac{1}{4 \eta}\right)\left\|\frac{\partial u}{\partial \nu}\right\|_{L^{2}\left(\Gamma_{1}\right)}^{2} \\
& \geq C\|u\|_{Z}^{2}
\end{aligned}
$$

for some constant $C>0$ as long as $\operatorname{Re}(\theta)>2 L+2 \eta K^{2}$ and $\eta$ is large enough.

So, recalling the Browder-Minty Theorem, if $\omega>2 L+2 \eta K^{2}$, the operator $A_{f}-\omega I$ will be maximal dissipative. From this fact, by the Lumer-Philips theorem, the operator $A_{f}$ generates a strongly continuous semigroup, and therefore Theorem 2.4 and Corollary 2.2 are proved.

\section{Local well-posedness of strong solutions}

The main goal of this section is to prove the local existence of solutions (Theorem 2.5) for the problem (2.15) at $H^{2}$-level for $N \leq 3$. We have proved that the linear model with a forcing function $f: \Omega \times(0, T) \rightarrow V$ given in (2.9) is well-posed in $V$ with appropriate control estimates of the solution map given in Theorem 4.2.

We set

$$
F(u)=-(\kappa+i \beta)|u|^{p-1} u+\gamma u .
$$

To acquire the estimates given in Theorem 4.2, we differentiate equation (2.15) in time in the distributional sense. In fact, let $w=u_{t}$, then

$$
\begin{cases}w_{t}-(\lambda+i \alpha) \Delta w=F_{t}(u, w) & \text { in } \Omega, \\ w=0 & \text { on } \Gamma_{0}, \\ \frac{\partial w}{\partial \nu}+w_{t}=0 & \text { on } \Gamma_{1}, \\ w(0)=w_{0} & \text { in } \Omega,\end{cases}
$$

where

$$
\begin{aligned}
F_{t}(u, w) & \equiv-(\kappa+i \beta)\left\{\frac{(p+1)}{2}|u|^{p-1} w+\frac{(p-1)}{2}|u|^{p-3} u^{2} \bar{w}\right\}+\gamma w \\
w_{0} & \equiv(\lambda+i \alpha) \Delta u_{0}-(\kappa+i \beta)\left|u_{0}\right|^{p-1} u_{0}+\gamma u_{0} .
\end{aligned}
$$

We will be looking at a (desirably unique) fixed point of the map

$$
K: C\left(0, T ; H^{2}(\Omega) \cap V \times V\right) \rightarrow C\left(0, T ; H^{2}(\Omega) \cap V \times V\right),
$$

defined by

$$
K\left(u^{\star}, w^{\star}\right)=(u, w)
$$

where $u$ satisfies $(2.15)$ with $F\left(u^{\star}\right)$ and $w$ satisfies $(6.2)$ with $F_{t}\left(u^{\star}, w^{\star}\right)$. Once such a fixed point is found, it is routine to show that $u$ and $u_{t}$ are strong solutions to (2.15).

In order to establish the existence of a suitable fixed point we need a priori estimates. We begin with some preliminary nonlinear estimates which will be useful both for local and global theory. 
Lemma 6.1. Let $F(u)$ and $F_{t}(u, w)$ be given by (6.1) and (6.3), respectively. Given $u \in H^{2}(\Omega) \cap V$ and $w \in V$,

(i) if $N \leq 3$ and $p \geq 1$, then

$$
\|F(u)\|_{H^{1}(\Omega)} \leq C\left(\|u\|_{L^{\infty}(\Omega)}^{p-1}+1\right)\|u\|_{H^{1}(\Omega)} \leq C\left(\|u\|_{H^{2}(\Omega)}^{p-1}+1\right)\|u\|_{H^{1}(\Omega)} ;
$$

(ii) if $N \leq 3$ and $p \geq 2$, then

$$
\|F(u)\|_{H^{2}(\Omega)} \leq\left(C\|u\|_{L^{\infty}(\Omega)}^{p-1}+1\right)\|u\|_{H^{2}(\Omega)} \leq\left(C\|u\|_{H^{2}(\Omega)}^{p-1}+1\right)\|u\|_{H^{2}(\Omega)} ;
$$

(iii) if $N \leq 3$ and $p \geq 2$, then

$$
\left\|F_{t}(u, w)\right\|_{V} \leq C\|\nabla w\|_{L^{2}(\Omega)}\left\{\|u\|_{H^{2}(\Omega)}^{p-1}+1\right\}
$$

(iv) if $N=1$ and $p \geq 2$, then

$$
\left\|F_{t}(u, w)\right\|_{V} \leq C\|w\|_{V}\left(1+\|u\|_{V}^{p-1}\right)
$$

(v) if $N=2$ and $p \geq 2$, then

$$
\left\|F_{t}(u, w)\right\|_{V} \leq C\|w\|_{V}\left(\|u\|_{H^{2}(\Omega)}^{\theta+\frac{p-2}{2}}\|u\|_{V}^{1-\theta}\|u\|_{2}^{\frac{p-2}{2}}+\|u\|_{H^{2}(\Omega)}^{\frac{p-1}{2}}\|u\|_{2}^{\frac{p-1}{2}}+1\right),
$$

where $1>\theta>0$ can be chosen small. Moreover, for $p \in[2,5]$ and small $\theta$, one has

$$
\left\|F_{t}(u, w)\right\|_{V} \leq C\|w\|_{V}\left(\|u\|_{H^{2}(\Omega)}^{2}+\|u\|_{V}^{\tau}+1\right)
$$

where $\tau=\tau(p, \theta)>0$;

(vi) if $N=3$ and $p \geq 2$, then

$$
\left\|F_{t}(u, w)\right\|_{V} \leq C\|w\|_{V}\left(\|u\|_{H^{2}(\Omega)}^{\theta+\frac{3(p-2)}{4}}\|u\|_{V}^{1-\theta}\|u\|_{2}^{\frac{p-2}{4}}+\|u\|_{H^{2}(\Omega)}^{\frac{3(p-1)}{4}}\|u\|_{2}^{\frac{p-1}{4}}+1\right),
$$

where $1>\theta>0$ can be chosen small. Moreover, for $p \in\left[2, \frac{11}{3}\right]$ and small $\theta$, one has

$$
\left\|F_{t}(u, w)\right\|_{V} \leq C\|w\|_{V}\left(\|u\|_{H^{2}(\Omega)}^{2}+\|u\|_{V}^{\tau}+1\right)
$$

where $\tau=\tau(p, \theta)>0$.

Proof. Note that

$$
\nabla F(u)=-(\kappa+i \beta)\left\{\frac{(p+1)}{2}|u|^{p-1} \nabla u+\frac{(p-1)}{2}|u|^{p-3} u^{2} \nabla \bar{u}\right\}+\gamma \nabla u .
$$

Therefore,

$$
|\nabla F(u)| \leq C\left(|u|^{p-1}|\nabla u|+|\nabla u|\right) .
$$

The inequality (6.4) as well as the second part of (6.5) follows directly from embedding $H^{2}(\Omega) \hookrightarrow L^{\infty}(\Omega)$ . The first inequality in (6.5) was proved in [47] in the case of $p=3$ and $N=2$. A similar proof also applies to the more general case given in this lemma. The idea is to use the Gagliardo-Nirenberg inequality

$$
\|u\|_{W^{1,4}(\Omega)} \leq\|u\|_{L^{\infty}}^{\frac{1}{2}}\|u\|_{H^{2}(\Omega)}^{\frac{1}{2}} .
$$


In order to prove inequality (6.6), we first estimate $\nabla F_{t}(u, w)$ :

$$
\left|\nabla F_{t}(u, w)\right| \leq C\left(|u|^{p-2}|\nabla u \| w|+|u|^{p-1}|\nabla w|+|\gamma||\nabla w|\right) .
$$

Setting $C:=C(\kappa, \beta, p)$, by the triangle inequality, we have

$$
\left\|F_{t}(u, w)\right\|_{V} \leq C\left\{\|u\|_{L^{\infty}(\Omega)}^{p-2}\|w \nabla u\|_{L^{2}(\Omega)}+\|u\|_{L^{\infty}(\Omega)}^{p-1}\|\nabla w\|_{L^{2}(\Omega)}+|\gamma|\|\nabla w\|_{L^{2}(\Omega)}\right\} .
$$

Now, we use $\|w \nabla u\|_{L^{2}(\Omega)} \leq\|w\|_{V}\|u\|_{H^{2}(\Omega)}$ and $H^{2}(\Omega) \hookrightarrow L^{\infty}(\Omega)$ to obtain (6.6).

If $N=1$, then using $H^{1}(\Omega) \hookrightarrow L^{\infty}(\Omega)$, we can easily obtain from (6.13) that

$$
\left\|F_{t}(u, w)\right\|_{V} \leq C\|w\|_{V}\left(1+\|u\|_{V}^{p-1}\right) .
$$

If $N=2$, then by using the Gagliardo-Nirenberg inequality

$$
\|\nabla u\|_{L^{s}(\Omega)} \leq\|u\|_{H^{2}(\Omega)}^{\theta}\|u\|_{V}^{1-\theta},
$$

where $s>2$ and $0<\theta=1-\frac{2}{s}<1$, and the Sobolev embedding $H^{1}(\Omega) \hookrightarrow L^{q}(\Omega)(q \geq 1)$, we obtain

$$
\begin{aligned}
\|w \nabla u\|_{L^{2}(\Omega)} & \leq C\|w\|_{L^{r}(\Omega)}\|\nabla u\|_{L^{s}(\Omega)} \\
& \leq C\|w\|_{V}\|u\|_{H^{2}(\Omega)}^{\theta}\|u\|_{V}^{1-\theta}
\end{aligned}
$$

where $r=\frac{2 s}{s-2}$. Moreover, for $N=2$, one has the Gagliardo-Nirenberg inequality:

$$
\|u\|_{\infty} \leq\|u\|_{H^{2}(\Omega)}^{\frac{1}{2}}\|u\|_{2}^{\frac{1}{2}}
$$

Therefore, from (6.13) we obtain

$$
\left\|F_{t}(u, w)\right\|_{V} \leq C\|w\|_{V}\left\{\|u\|_{H^{2}(\Omega)}^{\theta+\frac{p-2}{2}}\|u\|_{V}^{1-\theta}\|u\|_{2}^{\frac{p-2}{2}}+\|u\|_{H^{2}(\Omega)}^{\frac{p-1}{2}}\|u\|_{2}^{\frac{p-1}{2}}+1\right\} .
$$

Observe that in the above inequality, $\frac{p-1}{2} \leq 2$ if $p \leq 5$.

If $N=3$, we can again use the Gagliardo-Nirenberg inequality in the form

$$
\|\nabla u\|_{L^{3}(\Omega)} \leq\|u\|_{H^{2}(\Omega)}^{\frac{1}{3}}\|u\|_{V}^{\frac{2}{3}}
$$

and use the embedding $H^{1}(\Omega) \hookrightarrow L^{6}(\Omega)$ to obtain

$$
\begin{aligned}
\|w \nabla u\|_{L^{2}(\Omega)} & \leq C\|w\|_{L^{6}(\Omega)}\|\nabla u\|_{L^{3}(\Omega)} \\
& \leq C\|w\|_{V}\|u\|_{H^{2}(\Omega)}^{\theta}\|u\|_{V}^{1-\theta} .
\end{aligned}
$$

Moreover, for $N=3$, one has the Gagliardo-Nirenberg inequality:

$$
\|u\|_{\infty} \leq\|u\|_{H^{2}(\Omega)}^{\frac{3}{4}}\|u\|_{2}^{\frac{1}{4}}
$$

Therefore, we obtain

$$
\left\|F_{t}(u, w)\right\|_{V} \leq C\|w\|_{V}\left\{\|u\|_{H^{2}(\Omega)}^{\theta+\frac{3(p-2)}{4}}\|u\|_{V}^{1-\theta}\|u\|_{2}^{\frac{p-2}{4}}+\|u\|_{H^{2}(\Omega)}^{\frac{3(p-1)}{4}}\|u\|_{2}^{\frac{p-1}{4}}+1\right\} .
$$

Note that in the above case, $\frac{3(p-1)}{4} \leq 2$ if $p \leq \frac{11}{3}$.

Now, we take into account the following compatibility condition: 
Definition 6.1 (Compatibility Condition $(C C)$ ). We consider

$$
\frac{\partial u_{0}}{\partial \nu}+(\lambda+i \alpha) \Delta u_{0}-F\left(u_{0}\right)=0 \text { on } \Gamma_{1} .
$$

We also define the following spaces:

$$
X_{0}=\left\{\begin{array}{l}
\left(u_{0}, w_{0}\right) \in V \times V \\
w_{0}=(\lambda+i \alpha) \Delta u_{0}-F\left(u_{0}\right) \\
\Delta u_{0} \in V \\
u_{0} \text { satisfies CC (Definition 6.1) }
\end{array}\right.
$$

and the Banach space

$$
X_{T}=\left\{(u, w): u \in C\left[0, T ; H^{2}(\Omega) \cap H_{\Gamma_{0}}^{1}(\Omega)\right], w \in C\left[0, T ; H_{\Gamma_{0}}^{1}(\Omega)\right), u_{t}=w\right\} .
$$

We note that from the elliptic theory, we get

$$
\Delta u_{0} \in V \hookrightarrow H^{1}(\Omega) \text { and } \frac{\partial u_{0}}{\partial \nu}=-\left.w_{0}\right|_{\Gamma_{1}} \in H^{1 / 2}(\Gamma) .
$$

So, we have the implication: $\left(u_{0}, w_{0}\right) \in X_{0} \Rightarrow u_{0} \in H^{2}(\Omega)$.

Thus, it makes sense to define the following norms on $X_{0}$ and $X_{T}$ :

$$
\begin{gathered}
\|(u, w)\|_{X_{0}}^{2}=\|u\|_{H^{2}(\Omega)}^{2}+\|w\|_{V}^{2}, \\
\|(u, w)\|_{X_{T}}^{2}=\sup _{t \in[0, T]}\|u\|_{H^{2}(\Omega)}^{2}+\sup _{t \in[0, T]}\|w\|_{V}^{2} .
\end{gathered}
$$

We will prove Theorem 2.5 in several steps.

Step 1: Setting the map. We will restrict the map $K$ defined previously to a special complete metric space in order to obtain a contraction.

\section{Lemma 6.2. Let}

$$
Q_{T} \equiv\left\{\left(u^{*}, w^{*}\right) \in X_{T} \text { s.t. } u^{*}(0)=u_{0}\right\}
$$

Then, $Q_{T}$ is a non-empty complete metric space with the metric induced from the norm of $X_{T}$, i.e., with the metric given by

$$
d_{Q_{T}}\left(\left(u_{1}^{*}, w_{1}^{*}\right),\left(u_{2}^{*}, w_{2}^{*}\right)\right)=\sup _{t \in[0, T]}\left\|u_{1}^{*}-u_{2}^{*}\right\|_{H^{2}(\Omega)}^{2}+\sup _{t \in[0, T]}\left\|w_{1}^{*}-w_{2}^{*}\right\|_{H_{\Gamma_{0}}^{1}(\Omega)}^{2} .
$$

Proof. $\left(u_{0}, 0\right) \in Q_{T}$, hence $Q_{T}$ is nonempty. It is easy to see that $d_{Q_{T}}$ is a metric. Now, in order to show completeness of $Q_{T}$, take $\left(u_{n}^{*}, w_{n}^{*}\right) \in Q_{T}$ such that $\left(u_{n}^{*}, w_{n}^{*}\right) \rightarrow\left(u^{*}, w^{*}\right) \in X_{T}$. This means $u_{n}^{*}(0)=u_{0}$ and $\lim _{n}\left[\sup _{t \in[0, T]}\left\|u_{n}^{*}-u^{*}\right\|_{H^{2}(\Omega)}^{2}\right]=0$, which implies

$$
0 \leq\left\|u^{*}(0)-u_{0}\right\|_{H^{2}(\Omega)}=\left\|u^{*}(0)-u_{n}^{*}(0)\right\|_{H^{2}(\Omega)} \leq \sup _{[0, T]}\left\|u^{*}-u_{n}^{*}\right\|_{H^{2}(\Omega)} .
$$

Passing to the limit as $n \rightarrow \infty$, we get $u^{*}(0)=u_{0}$. Namely, $\left(u^{*}, w^{*}\right) \in Q_{T}$. That is $Q_{T}$ is closed. But closed subsets of complete spaces are complete. Therefore, $Q_{T}$ is complete since $X_{T}$ is complete. 
Now let $\left(u_{0}, w_{0}\right) \in X_{0}$. We consider the map $K\left(u^{\star}, w^{\star}\right)$, with $\left(u^{\star}, w^{\star}\right) \in Q_{T}$, which produces solutions $(u, w) \in C^{1}([0, T] ; V) \cap C([0, T] ; V)$ to the respective problems:

$$
\left\{\begin{array}{l}
u_{t}-(\lambda+i \alpha) \Delta u=F\left(u^{\star}\right), \text { in } \Omega \times \mathbb{R}_{+}, \\
u=0, \text { on } \Gamma_{0} \times \mathbb{R}_{+}, \\
\frac{\partial u}{\partial \nu}+(\lambda+i \alpha) \Delta u-F\left(u^{\star}\right)=0, \text { on } \Gamma_{1} \times \mathbb{R}_{+}, \\
u(0)=u_{0}, \text { in } \Omega
\end{array}\right.
$$

and

$$
\left\{\begin{array}{l}
w_{t}-(\lambda+i \alpha) \Delta w=F_{t}\left(u^{\star}, w^{\star}\right), \text { in } \Omega \times \mathbb{R}_{+}, \\
w=0, \text { on } \Gamma_{0} \times \mathbb{R}_{+}, \\
\frac{\partial w}{\partial \nu}+(\lambda+i \alpha) \Delta w-F_{t}\left(u^{\star}, w^{\star}\right)=0, \text { on } \Gamma_{1} \times \mathbb{R}_{+}, \\
w(0)=w_{0}, \text { in } \Omega .
\end{array}\right.
$$

We would like to give some important remarks at this point.

\section{Remark 6.1.}

(i) It is important to note that $u_{0}$ satisfies the necessary compatibility conditions since $\left(u^{*}, w^{*}\right)$ is taken from the specially constructed space $Q_{T}$ introduced in the previous lemma, which enforces the equality $F\left(u^{*}(0)\right)=F\left(u_{0}\right)$.

(ii) One should also observe that $K$ maps the elements of $Q_{T}$ again to the elements of $Q_{T}$. This follows from the linear theory and the fact that $(u, w)=K\left(u^{*}, w^{*}\right)$ is continuous on $[0, T]$ so that in particular $u(0)=u_{0}$, i.e., $(u, w) \in Q_{T}$.

Componentwise, the map $K\left(u^{\star}, w^{\star}\right)$ can be thought as the composition of

$$
\left\{\begin{array}{l}
K\left(u^{\star}, \cdot\right): u^{\star} \longmapsto F\left(u^{\star}\right) \longmapsto u \\
K\left(\cdot, w^{\star}\right): w^{\star} \longmapsto F_{t}\left(\cdot, w^{\star}\right) \longmapsto w .
\end{array}\right.
$$

These component maps can be found explicitly via the "boundary solver" introduced in Lemma 4.2 as follows:

$$
\begin{aligned}
K\left(u^{\star}, \cdot\right) & =u(t) \\
& =\left(e^{A t} u_{0}^{*}-\int_{0}^{t} e^{A(t-s)} A \mathcal{N} F\left(u^{\star}(s)\right) d s+\int_{0}^{t} e^{A(t-s)} F\left(u^{\star}(s)\right) d s\right) \\
K\left(\cdot, w^{\star}\right) & =w(t) \\
& =\left(e^{A t} w_{0}^{*}-\int_{0}^{t} e^{A(t-s)} A \mathcal{N} F_{t}\left(\cdot, w^{\star}(s)\right) d s+\int_{0}^{t} e^{A(t-s)} F_{t}\left(\cdot, w^{\star}(s)\right) d s\right) .
\end{aligned}
$$

As before, $A$ is the operator given in (2.4) with Wentzell boundary conditions, $\mathcal{N}$ is the Neumann map as seen in (4.1).

\section{Step 2: The estimates: Invariance of the Ball in $X_{T}$}

We recall that $K\left(u^{\star}, w^{\star}\right)=(u, w)$ where $u$ satisfies (6.18) with the right hand side $f \equiv F\left(u^{\star}\right)$ and $w$ satisfies (6.19) with the right hand side $f \equiv F_{t}\left(u^{\star}, w^{\star}\right)$. Since the initial data satisfies the required compatibility conditions, we are in a position to apply the estimates of Theorem 4.2 . This yields:

$$
\begin{aligned}
& \|w\|_{C([0, T] ; V)}+\|u\|_{C\left([0, T] ; H^{2}(\Omega)\right)} \\
& \leq C\left(\left\|F\left(u^{\star}\right)\right\|_{L^{2}(0, T ; V)}+\left\|F_{t}\left(u^{\star}, w^{\star}\right)\right\|_{L^{2}(0, T, V)}+\left\|u_{0}\right\|_{V}+\left\|\Delta u_{0}\right\|_{V}\right) .
\end{aligned}
$$


We first need to verify that $K\left(u^{\star}, w^{\star}\right)$ maps $B_{R}\left(Q_{T}\right)$ into $B_{R}\left(Q_{T}\right)$, where $B_{R}\left(Q_{T}\right)$ denotes the closed ball of radius $R$ in the space $Q_{T}$. Below we will suitably choose $R$ and $T$. To accomplish this, we shall use the estimates in (6.22) and Lemma 6.1. To this end, let $R>0$ be a fixed number (to be chosen in a moment) such that $\left(u^{*}, w^{*}\right) \in B_{R}\left(X_{T}\right)$.

Making use of estimates (6.4) and (6.6) given in Lemma 6.1, we conclude that

$$
\begin{aligned}
& \left\|F\left(u^{\star}\right)\right\|_{L^{2}(0, T ; V)}+\left\|F_{t}\left(u^{\star}, w^{\star}\right)\right\|_{L^{2}(0, T, V)} \\
& =\left[\int_{0}^{T}\left\|F\left(u^{\star}(t)\right)\right\|_{V}^{2} d t\right]^{1 / 2}+\left[\int_{0}^{T}\left\|F_{t}\left(u^{\star}(t), w^{\star}(t)\right)\right\|_{V}^{2} d t\right]^{1 / 2} \\
& \leq\left[\int_{0}^{T} 2\left(1+\left\|u^{\star}(t)\right\|_{H^{2}(\Omega)}^{2(p-1)}\right)\left(\left\|u^{\star}(t)\right\|_{V}^{2}+\left\|w^{\star}(t)\right\|_{V}^{2}\right) d t\right]^{1 / 2} \\
& \leq C T^{1 / 2}\left(1+\left\|u^{\star}\right\|_{C\left([0, T] ; H^{2}(\Omega)\right)}^{p-1}\right)\left(\left\|u^{\star}\right\|_{C\left([0, T] ; H^{2}(\Omega)\right)}+\left\|w^{\star}\right\|_{C([0, T] ; V)}\right) . \\
& \leq C 2 R T^{1 / 2}\left(1+R^{p-1}\right) .
\end{aligned}
$$

Combining (6.22) and (6.23), we infer,

$$
\begin{aligned}
\left\|K\left(u^{\star}, w^{\star}\right)\right\|_{X_{T}} & =\|(u, w)\|_{X_{T}} \\
& =\|w\|_{C([0, T] ; V)}+\|u\|_{C\left([0, T] ; H^{2}(\Omega)\right)} \\
& \leq C_{u_{0}}+C 2 R T^{1 / 2}\left(1+R^{p-1}\right) .
\end{aligned}
$$

Let $R=2 C_{u_{0}}$. Then, for small $T, K$ maps $B_{R}\left(Q_{T}\right)$ into itself.

Step 3: Contraction. We will show that for small $T$, there exist $1>\rho>0$ such that

$$
\left\|K\left(u_{1}^{\star}, w_{1}^{\star}\right)-K\left(u_{2}^{\star}, w_{2}^{\star}\right)\right\|_{X_{T}} \leq \rho\left\|\left(u_{1}^{\star}-u_{2}^{\star}, w_{1}^{\star}-w_{2}^{\star}\right)\right\|_{X_{T}}, \forall\left(u_{1}^{\star}, w_{1}^{\star}\right),\left(u_{2}^{\star}, w_{2}^{\star}\right) \in B_{R}\left(X_{T}\right) .
$$

Let $\left(u_{1}^{*}, w_{1}^{*}\right),\left(u_{2}^{*}, w_{2}^{*}\right) \in X_{T}$. Then by arguments similar to those above, we can obtain

$$
\begin{aligned}
\left\|K\left(u_{1}^{\star}, w_{1}^{\star}\right)-K\left(u_{2}^{\star}, w_{2}^{\star}\right)\right\|_{X_{T}} & =\left\|\left(u_{1}-u_{2}, w_{1}-w_{2}\right)\right\|_{X_{T}} \\
& =\left\|u_{1}-u_{2}\right\|_{C\left([0, T] ; H^{2}(\Omega)\right)}+\left\|w_{1}-w_{2}\right\|_{C([0, T] ; V)} \\
& \leq \int_{0}^{T}\left\|F\left(u_{1}^{\star}(s)\right)-F\left(u_{2}^{\star}(s)\right)\right\|_{H^{2}(\Omega)} d s \\
& +\int_{0}^{T}\left\|F_{t}\left(u_{1}^{\star}(s), w_{1}^{\star}(s)\right)-F_{t}\left(u_{2}^{\star}(s), w_{2}^{\star}(s)\right)\right\|_{V} d s .
\end{aligned}
$$

By using the local Lipschitz estimates for $F$ and $F_{t}$, taking into account that $\left(u_{1}^{\star}, w_{1}^{\star}\right),\left(u_{2}^{\star}, w_{2}^{\star}\right) \in B_{R}\left(X_{T}\right)$, one obtains:

$$
\begin{aligned}
\left\|F\left(u_{1}^{\star}\right)-F\left(u_{2}^{\star}\right)\right\|_{H^{2}(\Omega)} & \leq C_{1}\left(\left\|u_{1}^{\star}\right\|_{H^{2}(\Omega)}^{c(p)},\left\|u_{2}^{\star}\right\|_{H^{2}(\Omega)}^{c(p)},|\gamma|\right)\left\|u_{1}^{\star}-u_{2}^{\star}\right\|_{H^{2}(\Omega)} \\
& \leq C_{1}(R)\left\|u_{1}^{\star}-u_{2}^{\star}\right\|_{H^{2}(\Omega)}
\end{aligned}
$$

and

$$
\begin{aligned}
\left\|F_{t}\left(u_{1}^{\star}, w_{1}^{\star}\right)-F\left(u_{2}^{\star}, w_{2}^{\star}\right)\right\|_{V} & \leq C_{2}\left(\left\|u_{1}^{\star}\right\|_{H^{2}(\Omega)}^{c(p)},\left\|u_{2}^{\star}\right\|_{H^{2}(\Omega)}^{c(p)},|\gamma|\right)\left\|w_{1}^{\star}-w_{2}^{\star}\right\|_{V} \\
& \leq C_{2}(R)\left\|w_{1}^{\star}-w_{2}^{\star}\right\|_{V} .
\end{aligned}
$$

Setting $C_{3}:=\max \left\{C_{1}(R), C_{2}(R)\right\}$, from (6.24), we have

$$
\begin{aligned}
& \left\|K\left(u_{1}^{\star}, w_{1}^{\star}\right)-K\left(u_{2}^{\star}, w_{2}^{\star}\right)\right\|_{X_{T}} \\
& \leq C_{3} \int_{0}^{T}\left[\left\|u_{1}^{\star}(s)-u_{2}^{\star}(s)\right\|_{H^{2}(\Omega)}+\left\|w_{1}^{\star}(s)-w_{2}^{\star}(s)\right\|_{V}\right] d s \\
& \leq T C_{3}\left[\left\|u_{1}^{\star}-u_{2}^{\star}\right\|_{C\left([0, T] ; H^{2}(\Omega)\right)}+\left\|w_{1}^{\star}-w_{2}^{\star}\right\|_{C([0, T] ; V)}\right] \\
& \leq T C_{3}\left\|\left(u_{1}^{\star}-u_{2}^{\star}, w_{1}^{\star}-w_{2}^{\star}\right)\right\|_{X_{T}} .
\end{aligned}
$$


The above estimate proves that $K$ is a contraction if $T$ is sufficiently small. This proves the local existence and uniqueness of a strong solution in $B_{R}\left(Q_{T}\right)$.

Remark 6.2. The above result says that there is a unique local solution in $B_{R}\left(Q_{T}\right)$. This does not mean that there is uniqueness in $Q_{T}$. In the case, $\alpha, \beta>0$, one can also prove the uniqueness in $Q_{T}$. This directly follows from (7.10).

\section{Global well-posedness of strong solutions}

In this section, we study the global strong solutions of the CGLE with dynamic boundary conditions with power type nonlinearity under the additional assumption $\beta>0$. Our proofs for strong solutions will use Sobolev embeddings. Therefore, we will have some restriction on $p$. In [19], it is proven that the defocusing cubic NLS with dynamic boundary conditions is globally well-posed for $N=2$. Here, we improve this result in the context of the CGLE by proving the well-posedness of global solutions for dimensions $N \leq 3$. More precisely, we prove global well-posedness for $p \geq 2$ if $N=1 ; p \in[2,5]$ if $N=2$; and $p \in\left[2, \frac{11}{3}\right]$ if $N=3$. The smoothing effect will play a major role in this context. This is a missing ingredient in the case of the nonlinear Schrödinger equations.

Suppose $0<T_{\max } \leq \infty$ denotes the maximal time of existence of a given local strong solution. We want to prove that $T_{\max }=\infty$ by showing that $\left\|\left(u, u_{t}\right)\right\|_{X_{T}}$ remain bounded on $\left[0, T_{\max }\right)$. To this end, we first prove the following lemma which is true in any dimension. Similar estimates were proved, for example, in [2, Lemmas 3.2 and 5.2], where the domain was either the whole space or a torus (periodic boundary conditions). Here, our aim is to obtain a uniform energy bound for the solutions.

Lemma 7.1. Let $u_{0} \in V \cap L^{p+1}(\Omega)$ with $\gamma_{0} u_{0} \in L^{p+1}\left(\Gamma_{1}\right)$. Then,

(i) if $\gamma>0$, then $E(t) \leq E(0) \exp \left(C T_{\max }\right)$ for $t \in\left[0, T_{\max }\right)$, where

$$
\begin{aligned}
E(t) \equiv & \frac{\alpha}{2}\|\nabla u(t)\|_{L^{2}(\Omega)}^{2}+\frac{\beta}{p+1}\|u(t)\|_{L^{p+1}(\Omega)}^{p+1}+\frac{1}{p+1}(\alpha \kappa+\beta \lambda)\|u\|_{L^{p+1}\left(\Gamma_{1}\right)}^{p+1} \\
& +\alpha \int_{0}^{t}\left\|u_{t}(s)\right\|_{L^{2}\left(\Gamma_{1}\right)}^{2} d s+\alpha \lambda \int_{0}^{t}\|\Delta u\|_{L^{2}(\Omega)}^{2} d s+\kappa \beta \int_{0}^{t}\|u(s)\|_{L^{2 p}(\Omega)}^{2 p} .
\end{aligned}
$$

(ii) if $\gamma \leq 0$, then $E(t) \leq E(0)$ for $t \in\left[0, T_{\max }\right)$, where

$$
\begin{aligned}
E(t) \equiv & \frac{\alpha}{2}\|\nabla u(t)\|_{L^{2}(\Omega)}^{2}+\frac{\beta}{p+1}\|u(t)\|_{L^{p+1}(\Omega)}^{p+1}-\frac{\alpha \gamma}{2}\|u(t)\|_{L^{2}\left(\Gamma_{1}\right)}^{2}+\frac{1}{p+1}(\alpha \kappa+\beta \lambda)\|u\|_{L^{p+1}\left(\Gamma_{1}\right)}^{p+1} \\
& +\alpha \int_{0}^{t}\left\|u_{t}(s)\right\|_{L^{2}\left(\Gamma_{1}\right)}^{2} d s+\alpha \lambda \int_{0}^{t}\|\Delta u\|_{L^{2}(\Omega)}^{2} d s+\kappa \beta \int_{0}^{t}\|u(s)\|_{L^{2 p}(\Omega)}^{2 p} .
\end{aligned}
$$

Moreover, in both cases above, the solutions enjoy the following interior and boundary regularity for all $T>0$ :

$$
\begin{gathered}
\left(u, u_{t}\right) \in\left[C\left([0, T] ; V \cap L^{p+1}(\Omega)\right) \cap L^{2}\left(0, T ; H^{2}(\Omega)\right) \cap L^{2 p}\left(0, T ; L^{2 p}(\Omega)\right)\right] \times L^{2}\left(0, T ; L^{2}(\Omega)\right), \\
\frac{\partial u}{\partial n}=\gamma_{0} u_{t} \in L^{2}\left(0, T ; L^{2}\left(\Gamma_{1}\right)\right), \gamma_{0} u \in C\left([0, T] ; L^{p+1}\left(\Gamma_{1}\right)\right) .
\end{gathered}
$$

Proof. We start by taking the real part of the scalar product of $-\alpha \Delta u+\beta|u|^{p-1} u$ and $u_{t}$ :

$$
\begin{aligned}
& \operatorname{Re}\left(-\alpha \Delta u+\beta|u|^{p-1} u, u_{t}\right)=\operatorname{Re}\left(-\alpha \Delta u+\beta|u|^{p-1} u,(\lambda+i \alpha) \Delta u-(\kappa+i \beta)|u|^{p-1} u+\gamma u\right) \\
& =-\alpha \lambda\|\Delta u\|_{L^{2}(\Omega)}^{2}+\alpha \gamma\|\nabla u\|_{L^{2}(\Omega)}^{2}-\alpha \gamma \operatorname{Re}\left(\frac{\partial u}{\partial n}, u\right)_{L^{2}\left(\Gamma_{1}\right)}-\kappa \beta\|u\|_{L^{2 p}(\Omega)}^{2 p}+\beta \gamma\|u\|_{L^{p+1}(\Omega)}^{p+1} \\
& \quad+\alpha \operatorname{Re}\left(\Delta u,(\kappa+i \beta)|u|^{p-1} u\right)+\beta \operatorname{Re}\left(|u|^{p-1} u,(\lambda+i \alpha) \Delta u\right)
\end{aligned}
$$




$$
\begin{aligned}
= & -\alpha \lambda\|\Delta u\|_{L^{2}(\Omega)}^{2}+\alpha \gamma\|\nabla u\|_{L^{2}(\Omega)}^{2}-\alpha \gamma \operatorname{Re}\left(\frac{\partial u}{\partial n}, u\right)_{L^{2}\left(\Gamma_{1}\right)}-\kappa \beta\|u\|_{L^{2 p}(\Omega)}^{2 p}+\beta \gamma\|u\|_{L^{p+1}(\Omega)}^{p+1} \\
& -\alpha \operatorname{Re}\left(\nabla u,(\kappa+i \beta) \nabla\left(|u|^{p-1} u\right)\right)+\alpha \operatorname{Re}\left(\frac{\partial u}{\partial n},(\kappa+i \beta)|u|^{p-1} u\right)_{L^{2}\left(\Gamma_{1}\right)} \\
& -\beta \operatorname{Re}\left(\nabla\left(|u|^{p-1} u\right),(\lambda+i \alpha) \nabla u\right)+\beta \operatorname{Re}\left(|u|^{p-1} u,(\lambda+i \alpha) \frac{\partial u}{\partial n}\right)_{L^{2}\left(\Gamma_{1}\right)} .
\end{aligned}
$$

The first boundary term at the right hand side of (7.3) can be estimated by

$$
\begin{aligned}
\left|-\alpha \gamma \operatorname{Re}\left(\frac{\partial u}{\partial n}, u\right)_{L^{2}\left(\Gamma_{1}\right)}\right| & \leq \frac{\alpha \gamma}{2}\left(C_{\epsilon}\|u\|_{L^{2}\left(\Gamma_{1}\right)}^{2}+\epsilon\left\|\frac{\partial u}{\partial n}\right\|_{L^{2}\left(\Gamma_{1}\right)}^{2}\right) \\
& \leq \frac{\alpha \gamma}{2}\left(C_{\epsilon}\|\nabla u\|_{L^{2}(\Omega)}^{2}+\epsilon\left\|u_{t}\right\|_{L^{2}\left(\Gamma_{1}\right)}^{2}\right) .
\end{aligned}
$$

Alternatively, by using $\left.\frac{\partial u}{\partial \nu}\right|_{\Gamma_{1}}=-u_{t}$ one also has

$$
-\alpha \gamma R e\left(\frac{\partial u}{\partial n}, u\right)_{L^{2}\left(\Gamma_{1}\right)}=\frac{\alpha \gamma}{2} \frac{d}{d t}\|u\|_{L^{2}\left(\Gamma_{1}\right)}^{2} .
$$

Under the assumption $\beta>0$, we have $\alpha \kappa+\beta \lambda>0$ and

$$
\begin{aligned}
& -\alpha \operatorname{Re}\left(\nabla u,(\kappa+i \beta) \nabla\left(|u|^{p-1} u\right)\right)-\beta \operatorname{Re}\left(\nabla\left(|u|^{p-1} u\right),(\lambda+i \alpha) \nabla u\right) \\
& \quad=-(\alpha \kappa+\beta \lambda) \operatorname{Re}\left(\nabla\left(|u|^{p-1} u\right), \nabla u\right)=-(\alpha \kappa+\beta \lambda) \operatorname{Re}\left(\nabla u,\left(\frac{p+1}{2}|u|^{p-1} \nabla u+\frac{p-1}{2}|u|^{p-3} u^{2} \nabla \bar{u}\right)\right) \\
& \quad=-(\alpha \kappa+\beta \lambda)\left[\frac{p+1}{2} \int_{\Omega}|u|^{p-1}|\nabla u|^{2} d x+\frac{p-1}{2} \operatorname{Re} \int_{\Omega}|u|^{p-3} \bar{u}^{2}(\nabla u)^{2} d x\right] \leq 0 .
\end{aligned}
$$

The last term at the right hand side of $(7.3)$ is calculated as follows:

$$
\begin{aligned}
& \alpha \operatorname{Re}\left(\frac{\partial u}{\partial n},(\kappa+i \beta)|u|^{p-1} u\right)_{L^{2}\left(\Gamma_{1}\right)}+\beta \operatorname{Re}\left(|u|^{p-1} u,(\lambda+i \alpha) \frac{\partial u}{\partial n}\right)_{L^{2}\left(\Gamma_{1}\right)} \\
& \quad=(\alpha \kappa+\beta \lambda) \operatorname{Re}\left(|u|^{p-1} u, \frac{\partial u}{\partial n}\right)_{L^{2}\left(\Gamma_{1}\right)}=-\frac{1}{(p+1)}(\alpha \kappa+\beta \lambda) \frac{d}{d t}\|u\|_{L^{p+1}\left(\Gamma_{1}\right)}^{p+1}
\end{aligned}
$$

On the other hand, by using the main equation, we can rewrite the same scalar product in (7.3) as

$$
\begin{aligned}
\operatorname{Re}\left(-\alpha \Delta u+\beta|u|^{p-1} u, u_{t}\right) & =\frac{\alpha}{2} \frac{d}{d t}\|\nabla u\|_{L^{2}(\Omega)}^{2}+\frac{\beta}{p+1} \frac{d}{d t}\|u\|_{L^{p+1}(\Omega)}^{p+1}-\alpha \operatorname{Re}\left(\frac{\partial u}{\partial n}, u_{t}\right)_{L^{2}\left(\Gamma_{1}\right)} \\
& =\frac{\alpha}{2} \frac{d}{d t}\|\nabla u\|_{L^{2}(\Omega)}^{2}+\frac{\beta}{p+1} \frac{d}{d t}\|u\|_{L^{p+1}(\Omega)}^{p+1}+\alpha\left\|u_{t}\right\|_{L^{2}\left(\Gamma_{1}\right)}^{2} .
\end{aligned}
$$

If $\gamma \geq 0$, it follows that

$$
\begin{aligned}
E(t)-E(0) & \leq \alpha \gamma\left(1+\frac{C_{\epsilon}}{2}\right) \int_{0}^{t}\|\nabla u(s)\|_{L^{2}(\Omega)}^{2} d s+\beta \gamma \int_{0}^{t}\|u(s)\|_{L^{p+1}(\Omega)}^{p+1}+\epsilon \frac{\alpha \gamma}{2} \int_{0}^{t}\left\|u_{t}(s)\right\|_{L^{2}\left(\Gamma_{1}\right)}^{2} d s \\
& \leq \epsilon \frac{\gamma}{2} E(t)+C \int_{0}^{t} E(s) d s,
\end{aligned}
$$

where $\epsilon>0$ small and fixed.

Therefore,

$$
E(t) \leq C E(0)+C \int_{0}^{t} E(s) d s .
$$


Now, using Gronwall's inequality, we conclude that

$$
E(t) \leq E(0) \exp \left(C T_{\max }\right)
$$

for $t \in\left[0, T_{\max }\right)$.

Now, we consider the case $\gamma<0$ and define $E(t)$ as in (7.2). Bu utilizing the alternative calculation in (7.5), we get

$$
E(t)-E(0) \leq \frac{\alpha \gamma}{2} \int_{0}^{t}\|\nabla u(s)\|_{L^{2}(\Omega)}^{2} d s+\beta \gamma \int_{0}^{t}\|u(s)\|_{L^{p+1}(\Omega)}^{p+1} \leq 0 .
$$

This gives $E(t) \leq E(0)$ when $\gamma<0$.

Hence, we proved that the corresponding solution $\left(u, u_{t}\right)$ is global in the sense that, for all $T>0$,

$$
\left(u, u_{t}\right) \in\left[C\left([0, T] ; V \cap L^{p+1}(\Omega)\right) \cap L^{2}\left(0, T ; H^{2}(\Omega)\right) \cap L^{2 p}\left(0, T ; L^{2 p}(\Omega)\right)\right] \times L^{2}\left(0, T ; L^{2}(\Omega)\right)
$$

and

$$
\gamma_{0} u \in C\left([0, T] ; L^{p+1}\left(\Gamma_{1}\right)\right), \frac{\partial u}{\partial n}=\gamma_{0} u_{t} \in L^{2}\left(0, T ; L^{2}\left(\Gamma_{1}\right)\right) .
$$

Lemma 7.2. Let $u$ be a local solution. Then,

(1) If $N=1$ and $1<p<\infty$, then $\|F(u)\|_{V} \leq C$.

(2) If $N=2$ and $1<p<\infty$, then $\|F(u)\|_{V} \leq C+C\|u\|_{H^{2}(\Omega)}^{\theta}$ where $1>\theta>0$ can be chosen as small as we wish.

(3) If $N \geq 3$ and $1<p<\frac{N}{N-2}$, then $\|F(u)\|_{V} \leq C+C\|u\|_{H^{2}(\Omega)}^{\theta}$ where $1>\theta>0$ can be chosen as small as we wish.

(4) If $N=3$ and $p \geq 3$, then $\|F(u)\|_{V} \leq C+C\|u\|_{H^{2}(\Omega)}^{\frac{3(p-1)}{4}}$. If in addition $p<\frac{11}{3}$, then $\|F(u)\|_{V} \leq$ $C_{\epsilon}+\epsilon\|u\|_{H^{2}(\Omega)}^{2}$ for any fixed small $\epsilon>0$.

Proof. Note that

$$
\nabla F(u)=-(\kappa+i \beta)\left\{\frac{(p+1)}{2}|u|^{p-1} \nabla u+\frac{(p-1)}{2}|u|^{p-3} u^{2} \nabla \bar{u}\right\}+\gamma \nabla u .
$$

Therefore,

$$
|\nabla F(u)| \leq C\left(|u|^{p-1}|\nabla u|+|\nabla u|\right) .
$$

The case $N=1$ follows from the Sobolev embedding $H^{1}(\Omega) \hookrightarrow L^{\infty}(\Omega)$ and Lemma 7.1.

Let $N=2$ and $1<p<\infty$. Then, by using the Gagliardo-Nirenberg inequality

$$
\|\nabla u\|_{L^{s}(\Omega)} \leq\|u\|_{H^{2}(\Omega)}^{\theta}\|u\|_{V}^{1-\theta},
$$

where $s>2$ and $0<\theta=1-\frac{2}{s}<1$, the Sobolev embedding $H^{1}(\Omega) \hookrightarrow L^{q}(\Omega)(q \geq 1)$, and Lemma 7.1, we have

$$
\|F(u)\|_{V} \leq C\left(\|u\|_{q}^{(p-1)}\|\nabla u\|_{s}+\|\nabla u\|_{L^{2}(\Omega)}\right) \leq C\left(\|u\|_{V}^{(p-1)}\|u\|_{V}^{1-\theta}\|u\|_{H^{2}(\Omega)}^{\theta}+\|u\|_{V}\right)
$$

with $q>2(p-1)$ and $q \geq 1$.

Let $N \geq 3$ and $1<p<\frac{N}{N-2}$; then we have the following Gagliardo-Nirenberg inequality:

$$
\|\nabla u\|_{L^{s}(\Omega)} \leq\|u\|_{H^{2}(\Omega)}^{\theta}\|u\|_{V}^{1-\theta}
$$

where $\frac{2 N}{N-2}>s=\frac{4 N}{2 N-2(N-2)(p-1)}>2$ and $0<\theta=\frac{(s-2) N}{2 s}<1$. 
For $q=\frac{2 N}{N-2},(7.13)$ can be rewritten as

$$
\begin{aligned}
\|F(u)\|_{V} & \leq C\left(\left\||u|^{p-1}|\nabla u|\right\|_{L^{2}(\Omega)}+|\nabla u| \|_{L^{2}(\Omega)}\right) \leq C\left(\|u\|_{q}^{(p-1)}\|\nabla u\|_{s}+\|u\|_{V}\right) \\
& \leq C\left(\|u\|_{V}^{(p-1)}\|u\|_{H^{2}(\Omega)}^{\theta}\|u\|_{V}^{1-\theta}+\|u\|_{V}\right) .
\end{aligned}
$$

Let $N=3$ and $p \geq 3$, then we have the Gagliardo-Nirenberg inequality

$$
\|u\|_{\infty} \leq C\|u\|_{H^{2}(\Omega)}^{\frac{3}{4}}\|u\|_{2}^{\frac{1}{4}} .
$$

Therefore,

$$
\|F(u)\|_{V} \leq C\left(\|u\|_{\infty}^{p-1}\|\nabla u\|_{L^{2}(\Omega)}+\|\nabla u\|_{L^{2}(\Omega)}\right) \leq C\left(\|u\|_{H^{2}(\Omega)}^{\frac{3}{4}(p-1)}\|u\|_{L^{2}(\Omega)}^{\frac{p-1}{4}}\|\nabla u\|_{2}+\|u\|_{V}\right) .
$$

Note that if in addition, $p<\frac{11}{3}$, then $\frac{3}{4}(p-1)<2$.

Now, we will prove the following estimate:

$$
\begin{aligned}
\left\|u_{t}(t)\right\|_{V}+\|u(t)\|_{H^{2}(\Omega)} \leq C\left(u_{0}, T_{\max }\right) & +C\left(\int_{0}^{t}\left\|F_{t}\left(u(s), u_{t}(s)\right)\right\|_{V} d s\right. \\
+ & {\left.\left[\int_{0}^{t} \int_{\Gamma_{1}}\left|F_{t}\left(u(s), u_{t}(s)\right)\right|^{2} d \Gamma d s\right]^{1 / 2}\right) }
\end{aligned}
$$

where the constants $C\left(u_{0}, T_{\max }\right)$ and $C$ are given more precisely later.

Substituting $f=F(u)$ in (2.9), using $g=-\left.f\right|_{\Gamma_{1}}$ and (4.18), (4.19) can be written as

$$
\begin{aligned}
u_{t}= & (\lambda+i \alpha) e^{A t} \triangle u_{0}+e^{A t} F\left(u_{0}\right)+\int_{0}^{t} e^{A(t-s)} F_{t}\left(u(s), u_{t}(s)\right) d s \\
& -A \int_{0}^{t} e^{A(t-s)} \mathcal{N} F_{t}\left(u(s), u_{t}(s)\right) d s .
\end{aligned}
$$

On the other hand, employing Lemma 7.2, it follows that

$$
\left\|e^{A t} F\left(u_{0}\right)\right\|_{V} \leq C\left(\left\|u_{0}\right\|_{H^{2}(\Omega)}\right) .
$$

Then, combining (7.18), (7.19), and the continuity of the map $\mathcal{L}$ given in (4.15) (whose bound, say is $\eta$ ), we obtain

$$
\begin{aligned}
\left\|u_{t}(t)\right\|_{V} & \leq \underbrace{C\left(\left\|\Delta u_{0}\right\|_{V},\left\|u_{0}\right\|_{H^{2}(\Omega)}\right)}_{:=C_{u_{0}}}+\int_{0}^{t}\left\|F_{t}\left(u(s), u_{t}(s)\right)\right\|_{V} d s+\left\|\mathcal{L}\left(F_{t}\left(u(s), u_{t}(s)\right)\right)\right\|_{C([0, T] ; V)} \\
& \leq C_{u_{0}}+\int_{0}^{t}\left\|F_{t}\left(u(s), u_{t}(s)\right)\right\|_{V} d s+\eta\left\|F_{t}\left(u(s), u_{t}(s)\right)\right\|_{L^{2}\left(0, t ; L^{2}\left(\Gamma_{1}\right)\right)} \\
& =C_{u_{0}}+\int_{0}^{t}\left\|F_{t}\left(u(s), u_{t}(s)\right)\right\|_{V} d s+\eta\left[\int_{0}^{t}\left\|F_{t}\left(u(s), u_{t}(s)\right)\right\|_{L^{2}\left(\Gamma_{1}\right)}^{2} d s\right]^{1 / 2} \\
& \leq C_{u_{0}}+\int_{0}^{t}\left\|F_{t}\left(u(s), u_{t}(s)\right)\right\|_{V} d s+\eta\left[\int_{0}^{t}\left\|F_{t}\left(u(s), u_{t}(s)\right)\right\|_{L^{2}\left(\Gamma_{1}\right)}^{2} d s\right]^{1 / 2} .
\end{aligned}
$$

Now, from (4.21) and the continuity of the trace map $\gamma: H^{1}(\Omega) \rightarrow H^{1 / 2}\left(\Gamma_{1}\right)$ (whose embedding constant, say $\varsigma$ ), we have

$$
\begin{aligned}
\|u(t)\|_{H^{2}(\Omega)} & \leq C_{1}\left(\left\|u_{t}(t)\right\|_{V}+\|F(u(t))\|_{V}+\|F(u(t))\|_{H^{1 / 2}\left(\Gamma_{1}\right)}\right) \\
& \leq C_{1}\left(\left\|u_{t}(t)\right\|_{V}+(1+\varsigma)\|F(u(t))\|_{V}\right) .
\end{aligned}
$$

Thus, combining Lemma 7.2, (7.20) and (7.21), (7.17) follows. 
Now, we employ an inequality derived from the Gagliardo-Nirenberg inequality (see [2]):

$$
\begin{aligned}
\|z(t)\|_{H^{2}(\Omega)} & \leq C\left(\|z\|_{L^{2}(\Omega)}+\|\triangle z\|_{L^{2}(\Omega)}\right) \\
& \leq \widetilde{C}\left(\|z\|_{V}+\|\triangle z\|_{L^{2}(\Omega)}\right), \forall z \in H^{2}(\Omega) .
\end{aligned}
$$

On the other hand, from Lemma 6.1 and (7.22), we obtain:

$$
\int_{0}^{t}\left\|F_{t}\left(u(s), u_{t}(s)\right)\right\|_{V} d s \leq C \int_{0}^{t}\|w(s)\|_{V}\left(\|u(s)\|_{H^{2}(\Omega)}^{2}+1\right) d s .
$$

Having in mind that $\widetilde{\gamma}$ denotes a trace operator-restriction to the boundary $\Gamma_{1}$, a mixed trace-interpolation inequality results

$$
\|\widetilde{\gamma} u\|_{L^{2}\left(\Gamma_{1}\right)}^{2} \leq C\|u\|_{H^{1}(\Omega)}\|u\|_{L^{2}(\Omega)} .
$$

This implies

$$
\left\|\widetilde{\gamma} F_{t}(u, w)\right\|_{L^{2}\left(\Gamma_{1}\right)}^{2} \leq C\left\|F_{t}(u, w)\right\|_{H^{1}(\Omega)}\left\|F_{t}(u, w)\right\|_{L^{2}(\Omega)} \cdot
$$

Recall that for $N=1$, we have $H^{1}(\Omega) \hookrightarrow L^{\infty}(\Omega)$; for $N=2, H^{1}(\Omega) \hookrightarrow L^{q}(\Omega)$ if $1 \leq q<\infty$; for $N \geq 3$, $H^{1}(\Omega) \hookrightarrow L^{q}(\Omega)$ if $1 \leq q \leq \frac{2 N}{N-2}$. Using these Sobolev embeddings and Lemma 7.1, for $p \leq \frac{N}{N-2}$ if $N \geq 3$ we get:

$$
\left\|F_{t}(u, w)\right\|_{L^{2}(\Omega)} \leq C\left(\left\||u|^{p-1} w\right\|_{L^{2}(\Omega)}+\|w\|_{L^{2}(\Omega)}\right) \leq C E(0)\|w\|_{V}
$$

Lemma 7.3. Let $\alpha, \beta, \lambda, \kappa>0$. Then, there exists $M>0$ such that

$$
\sup _{t \in\left[0, T_{\max }\right)}\|w(t)\|_{V}<M<\infty
$$

Proof. Multiply, (6.2) by $-\alpha \Delta \bar{w}$, integrate over $\Omega$ and take the real parts. Then, by also using (7.26) we have

$$
\begin{aligned}
& \frac{\alpha}{2} \frac{d}{d t}\|\nabla w\|_{L^{2}(\Omega)}^{2}+\alpha\left\|w_{t}\right\|_{L^{2}\left(\Gamma_{1}\right)}^{2}+\alpha \lambda\|\Delta w\|_{2}^{2}=-\alpha \operatorname{Re}\left(F_{t}(u, w), \Delta w\right)_{L^{2}(\Omega)} \\
& \quad \leq \frac{1}{4 \epsilon}\left\|F_{t}(u, w)\right\|_{L^{2}(\Omega)}^{2}+\epsilon\|\Delta w\|_{L^{2}(\Omega)}^{2} \leq C(E(0))\|w\|_{V}^{2}+\epsilon\|\Delta w\|_{L^{2}(\Omega)}^{2} .
\end{aligned}
$$

The above inequality gives

$$
\|w(t)\|_{V}^{2} \leq\left\|w_{0}\right\|_{V}^{2}+C(E(0)) \int_{0}^{t}\|w(s)\|_{V}^{2} d s .
$$

Now, from Gronwall's inequality the desired property follows.

Remark 7.1. From (7.27), we note that we are able to control the term $\epsilon\|\Delta w\|_{L^{2}(\Omega)}^{2}$ with the term $\alpha \lambda\|\Delta w\|_{2}^{2}$ at the left hand side. This is an important property for CGLE. For instance, in the case of the nonlinear Schrödinger equation [34], one does not have such a control since $\lambda=0$.

Now, it follows from (7.25) and (7.26) that

$$
\left\|\widetilde{\gamma} F_{t}(u, w)\right\|_{L^{2}\left(\Gamma_{1}\right)}^{2} \leq C\left\|F_{t}(u, w)\right\|_{H^{1}(\Omega)} .
$$


Moreover, from (7.23), we get

$$
\int_{0}^{t}\left\|F_{t}\left(u(s), u_{t}(s)\right)\right\|_{V} d s \leq C+C \int_{0}^{t}\|u(s)\|_{H^{2}(\Omega)}^{2} d s .
$$

But the right hand side of (7.29) is bounded by a constant due to Lemma 7.1 (since $\lambda>0$ ). Combining this with (7.17), we obtain

$$
\left\|\left(u, u_{t}\right)\right\|_{X_{T}} \leq C .
$$

Hence, we obtained a uniform bound for $\left\|\left(u, u_{t}\right)\right\|_{X_{T}}$ on $\left[0, T_{\max }\right)$. The proof of the global existence of strong solutions is complete.

\section{Weak solutions}

In this section, we will prove the global existence and uniqueness of weak solutions given in Theorem 2.7. So, let

$$
u_{0} \in V \text { such that } \gamma_{0} \varphi \in L^{p+1}\left(\Gamma_{1}\right)
$$

and

$$
\left\{u_{\mu, 0}\right\}
$$

be smooth enough that

$$
u_{\mu, 0} \longrightarrow u_{0} \quad \text { in } Q
$$

and for each $\mu \in \mathbb{N}, u_{\mu}$ is the unique strong solution of (2.15) with initial data $\left\{u_{\mu, 0}\right\}$. Then, $u_{\mu}$ solves

$$
\begin{cases}\partial_{t} u_{\mu}-(\lambda+i \alpha) \triangle u_{\mu}+(\kappa+i \beta)\left|u_{\mu}\right|^{p-1} u_{\mu}-\gamma u_{\mu}=0 & \text { in } \Omega \times \mathbb{R}_{+} \\ \frac{\partial u_{\mu}}{\partial \nu}=-\partial_{t} u_{\mu} & \text { on } \Gamma_{1} \times \mathbb{R}_{+} \\ u_{\mu}=0 & \text { on } \Gamma_{0} \times \mathbb{R}_{+} \\ u_{\mu}(0)=u_{\mu, 0} & \text { in } \Omega .\end{cases}
$$

Now, we define $z_{\mu, \sigma}:=u_{\mu}-u_{\sigma}$ for $\mu, \sigma \in \mathbb{N}$. We prove the following for $\left\{z_{\mu, \sigma}\right\}$ :

Lemma 8.1. $E_{\mu, \sigma}(t) \leq E_{\mu, \sigma}(0) \exp (C T), t \in[0, T]$, where the definition of $E_{\mu, \sigma}$ is given in (8.43).

Proof. Setting $G\left(z_{\mu, \sigma}\right):=\left|u_{\mu}\right|^{p-1} u_{\mu}-\left|u_{\sigma}\right|^{p-1} u_{\sigma}$, we observe that

$$
\begin{aligned}
\operatorname{Re}( & \left.-\alpha \Delta z_{\mu, \sigma}+\beta G\left(z_{\mu, \sigma}\right), z_{\mu, \sigma}^{\prime}\right) \\
= & \operatorname{Re}\left(-\alpha \Delta z_{\mu, \sigma}+\beta G\left(z_{\mu, \sigma}\right),(\lambda+i \alpha) \Delta z_{\mu, \sigma}-(\kappa+i \beta) G\left(z_{\mu, \sigma}\right)+\gamma z_{\mu, \sigma}\right) \\
= & -\alpha \lambda\left\|\Delta z_{\mu, \sigma}\right\|_{L^{2}(\Omega)}^{2}+\alpha \gamma\left\|\nabla z_{\mu, \sigma}\right\|_{L^{2}(\Omega)}^{2}-\alpha \gamma \operatorname{Re}\left(\frac{\partial z_{\mu, \sigma}}{\partial n}, z_{\mu, \sigma}\right)_{L^{2}\left(\Gamma_{1}\right)} \\
& -\kappa \beta\left\|G\left(z_{\mu, \sigma}\right)\right\|_{2}^{2}+\beta \gamma\left(G\left(z_{\mu, \sigma}\right), z_{\mu, \sigma}\right) \\
& +\alpha \operatorname{Re}\left(\Delta z_{\mu, \sigma},(\kappa+i \beta) G\left(z_{\mu, \sigma}\right)\right)+\beta \operatorname{Re}\left(G\left(z_{\mu, \sigma}\right),(\lambda+i \alpha) \Delta z_{\mu, \sigma}\right) \\
= & -\alpha \lambda\left\|\Delta z_{\mu, \sigma}\right\|_{L^{2}(\Omega)}^{2}+\alpha \gamma\left\|\nabla z_{\mu, \sigma}\right\|_{L^{2}(\Omega)}^{2}-\alpha \gamma \operatorname{Re}\left(\frac{\partial z_{\mu, \sigma}}{\partial n}, z_{\mu, \sigma}\right)_{L^{2}\left(\Gamma_{1}\right)} \\
& -\kappa \beta\|f(u)\|_{2}^{2}+\beta \gamma\left(G\left(z_{\mu, \sigma}\right), z_{\mu, \sigma}\right) \\
& -\alpha \operatorname{Re}\left(\nabla z_{\mu, \sigma},(\kappa+i \beta) \nabla G\left(z_{\mu, \sigma}\right)\right)+\alpha \operatorname{Re}\left(\frac{\partial z_{\mu, \sigma}}{\partial n},(\kappa+i \beta) G\left(z_{\mu, \sigma}\right)\right)_{L^{2}\left(\Gamma_{1}\right)} \\
& -\beta \operatorname{Re}\left(\nabla G\left(z_{\mu, \sigma}\right),(\lambda+i \alpha) \nabla z_{\mu, \sigma}\right)+\beta \operatorname{Re}\left(G\left(z_{\mu, \sigma}\right),(\lambda+i \alpha) \frac{\partial z_{\mu, \sigma}}{\partial n}\right)_{L^{2}\left(\Gamma_{1}\right)} .
\end{aligned}
$$


On the other hand, it follows that

$$
\begin{aligned}
& \left|-\alpha \gamma \operatorname{Re}\left(\frac{\partial z_{\mu, \sigma}}{\partial n}, z_{\mu, \sigma}\right)_{L^{2}\left(\Gamma_{1}\right)}\right| \leq \frac{\alpha \gamma}{2}\left|z_{\mu, \sigma}^{\prime},\left(z_{\mu, \sigma}\right)_{L^{2}\left(\Gamma_{1}\right)}\right| \\
& \quad \leq \alpha \gamma M\left\|z_{\mu, \sigma}^{\prime}\right\|_{L^{2}\left(\Gamma_{1}\right)}\left\|z_{\mu, \sigma}\right\|_{L^{2}\left(\Gamma_{1}\right)} \\
& \quad \leq \frac{\alpha \gamma}{2}\left(C_{\epsilon_{1}}\left\|z_{\mu, \sigma}\right\|_{L^{2}\left(\Gamma_{1}\right)}^{2}+\epsilon_{1}\left\|z_{\mu, \sigma}^{\prime}\right\|_{L^{2}\left(\Gamma_{1}\right)}^{2}\right) \\
& \quad \leq \frac{\alpha \gamma}{2}\left(C_{\epsilon_{1}}\left\|\nabla z_{\mu, \sigma}\right\|_{L^{2}(\Omega)}^{2}+\epsilon_{1}\left\|z_{\mu, \sigma}^{\prime}\right\|_{L^{2}\left(\Gamma_{1}\right)}^{2}\right) .
\end{aligned}
$$

Alternatively, by using $\left.\frac{\partial z_{\mu, \sigma}}{\partial \nu}\right|_{\Gamma_{1}}=-z_{\mu, \sigma}^{\prime}$ one also has

$$
\begin{aligned}
&-\alpha \gamma \operatorname{Re}\left(\frac{\partial z_{\mu, \sigma}}{\partial n}, z_{\mu, \sigma}\right)_{L^{2}\left(\Gamma_{1}\right)}=\frac{\alpha \gamma}{2} \frac{d}{d t}\left\|z_{\mu, \sigma}\right\|_{L^{2}\left(\Gamma_{1}\right)}^{2} . \\
& \operatorname{Re}\left(-\alpha \Delta z_{\mu, \sigma}+\beta G\left(z_{\mu, \sigma}\right), z_{\mu, \sigma}^{\prime}\right)= \frac{\alpha}{2} \frac{d}{d t}\left\|\nabla z_{\mu, \sigma}\right\|_{L^{2}(\Omega)}^{2}-\operatorname{Re}\left(\frac{\partial z_{\mu, \sigma}}{\partial n}, z_{\mu, \sigma}^{\prime}\right)_{L^{2}\left(\Gamma_{1}\right)} \\
&+\operatorname{Re}\left(\beta G\left(z_{\mu, \sigma}\right), z_{\mu, \sigma}^{\prime}\right) \\
&= \frac{\alpha}{2} \frac{d}{d t}\left\|\nabla z_{\mu, \sigma}\right\|_{L^{2}(\Omega)}^{2}+\alpha\left\|z_{\mu, \sigma}^{\prime}\right\|_{L^{2}\left(\Gamma_{1}\right)}^{2}+\operatorname{Re}\left(\beta G\left(z_{\mu, \sigma}\right), z_{\mu, \sigma}^{\prime}\right) .
\end{aligned}
$$

Now, taking account of (7.22), from the following local Lipschitz estimates:

$$
\begin{aligned}
\left\|G\left(z_{\mu, \sigma}\right)\right\|_{L^{2}(\Omega)} & \leq\left(\left\|u_{\mu}\right\|_{H^{2}},\left\|u_{\sigma}\right\|_{H^{2}}\right)\left\|z_{\mu, \sigma}\right\|_{L^{2}(\Omega)} \leq C\left(\left\|u_{\mu, 0}\right\|_{H^{2}},\left\|u_{\sigma, 0}\right\|_{H^{2}}\right)\left\|z_{\mu, \sigma}\right\|_{L^{2}(\Omega)} \\
\left\|G\left(z_{\mu, \sigma}\right)\right\|_{V} & \leq\left(\left\|u_{\mu}\right\|_{H^{2}},\left\|u_{\sigma}\right\|_{H^{2}}\right)\left\|z_{\mu, \sigma}\right\|_{V} \leq C\left(\left\|u_{\mu, 0}\right\|_{H^{2}},\left\|u_{\sigma, 0}\right\|_{H^{2}}\right)\left\|z_{\mu, \sigma}\right\|_{V},
\end{aligned}
$$

we have

$$
\begin{aligned}
& \operatorname{Re}\left(-\alpha \Delta z_{\mu, \sigma}+\beta G\left(z_{\mu, \sigma}\right), z_{\mu, \sigma}^{\prime}\right)= \frac{\alpha}{2} \frac{d}{d t}\left\|\nabla z_{\mu, \sigma}\right\|_{L^{2}(\Omega)}^{2}+\alpha\left\|z_{\mu, \sigma}^{\prime}\right\|_{L^{2}\left(\Gamma_{1}\right)}^{2}+\operatorname{Re}\left(\beta G\left(z_{\mu, \sigma}\right), z_{\mu, \sigma}^{\prime}\right) \\
& \leq \frac{\alpha}{2} \frac{d}{d t}\left\|\nabla z_{\mu, \sigma}\right\|_{L^{2}(\Omega)}^{2}+\alpha\left\|z_{\mu, \sigma}^{\prime}\right\|_{L^{2}\left(\Gamma_{1}\right)}^{2} \\
&+\beta C^{\prime}\left\|\nabla G\left(z_{\mu, \sigma}\right)\right\|_{L^{2}(\Omega)}\left\|z_{\mu, \sigma}^{\prime}\right\|_{L^{2}(\Omega)} \\
& \leq \frac{\alpha}{2} \frac{d}{d t}\left\|\nabla z_{\mu, \sigma}\right\|_{L^{2}(\Omega)}^{2}+\alpha\left\|z_{\mu, \sigma}^{\prime}\right\|_{L^{2}\left(\Gamma_{1}\right)}^{2}+\beta C_{\epsilon_{2}}\left\|\nabla z_{\mu, \sigma}\right\|_{L^{2}(\Omega)}^{2} \\
&+\beta C \epsilon_{2}\left[\left\|\triangle z_{\mu, \sigma}\right\|_{L^{2}(\Omega)}^{2}+\left\|G\left(z_{\mu, \sigma}\right)\right\|_{L^{2}(\Omega)}^{2}+\left\|z_{\mu, \sigma}\right\|_{V}^{2}\right] \\
&-\alpha \operatorname{Re}\left(\nabla z_{\mu, \sigma},(\kappa+i \beta) \nabla G\left(z_{\mu, \sigma}\right)\right)-\beta \operatorname{Re}\left(\nabla G\left(z_{\mu, \sigma}\right),(\lambda+i \alpha) \nabla z_{\mu, \sigma}\right) \\
&=-(\alpha \kappa+\beta \lambda) \operatorname{Re}\left(\nabla\left(G\left(z_{\mu, \sigma}\right)\right), \nabla z_{\mu, \sigma}\right) \\
& \leq|\alpha \kappa+\beta \lambda| \operatorname{Re}\left(\nabla G\left(z_{\mu, \sigma}\right), \nabla z_{\mu, \sigma}\right) \\
& \leq|\alpha \kappa+\beta \lambda|\left\|G\left(z_{\mu, \sigma}\right)\right\|_{V}\left\|z_{\mu, \sigma}\right\|_{V} \leq C_{1}\left\|z_{\mu, \sigma}\right\|_{V}^{2}
\end{aligned}
$$

and

$$
\begin{gathered}
\left|\beta \gamma\left(G\left(z_{\mu, \sigma}\right), z_{\mu, \sigma}\right)\right| \leq \beta \gamma C_{2}\left\|z_{\mu, \sigma}\right\|_{V}^{2} . \\
\alpha \operatorname{Re}\left(\frac{\partial z_{\mu, \sigma}}{\partial n},(\kappa+i \beta) G\left(z_{\mu, \sigma}\right)\right)_{L^{2}\left(\Gamma_{1}\right)}+\beta \operatorname{Re}\left(G\left(z_{\mu, \sigma}\right),(\lambda+i \alpha) \frac{\partial z_{\mu, \sigma}}{\partial n}\right)_{L^{2}\left(\Gamma_{1}\right)} \\
=(\alpha \kappa+\beta \lambda) \operatorname{Re}\left(G\left(z_{\mu, \sigma}\right), \frac{\partial z_{\mu, \sigma}}{\partial n}\right)_{L^{2}\left(\Gamma_{1}\right)}{ }^{\leq}|\alpha \kappa+\beta \lambda|\left(C_{\epsilon_{3}}\left\|G\left(z_{\mu, \sigma}\right)\right\|_{L^{2}\left(\Gamma_{1}\right)}^{2}+\epsilon_{3}\left\|z_{\mu, \sigma}^{\prime}\right\|_{L^{2}\left(\Gamma_{1}\right)}^{2}\right) \\
\leq|\alpha \kappa+\beta \lambda|\left(C_{\epsilon_{3}}\left\|\nabla z_{\mu, \sigma}\right\|_{L^{2}(\Omega)}^{2}+\epsilon_{3}\left\|z_{\mu, \sigma}^{\prime}\right\|_{L^{2}\left(\Gamma_{1}\right)}^{2}\right) .
\end{gathered}
$$


Collecting all the estimates given above, we get

$$
\begin{aligned}
& \frac{\alpha}{2} \frac{d}{d t}\left\|\nabla z_{\mu, \sigma}\right\|_{L^{2}(\Omega)}^{2}+\alpha\left(1-\frac{\gamma \epsilon_{1}}{2}-|\alpha \kappa+\beta \lambda| \epsilon_{3}\right)\left\|z_{\mu, \sigma}^{\prime}\right\|_{L^{2}\left(\Gamma_{1}\right)}^{2} \\
& \quad+\left(\alpha \lambda-\beta C \epsilon_{2}\right)\left\|\Delta z_{\mu, \sigma}\right\|_{L^{2}(\Omega)}^{2}+\left(\kappa \beta-\beta C \epsilon_{2}\right)\left\|G\left(z_{\mu, \sigma}\right)\right\|_{2}^{2} \\
& \quad \leq C\left\|\nabla z_{\mu, \sigma}\right\|_{L^{2}(\Omega)}^{2} .
\end{aligned}
$$

Considering $\epsilon_{i}, i=1,2,3$ small enough and integrating (8.42) in $t \in[0, T]$, we have

$$
E_{\mu, \sigma}(t) \leq C E_{\mu, \sigma}(0)+C \int_{0}^{t} E_{\mu, \sigma}(s) d s
$$

where

$$
E_{\mu, \sigma}(t):=\left\|\nabla z_{\mu, \sigma}(t)\right\|_{L^{2}(\Omega)}^{2}+C_{1} \int_{0}^{t}\left\|z_{\mu, \sigma}^{\prime}(s)\right\|_{L^{2}\left(\Gamma_{1}\right)}^{2} d s+C_{2} \int_{0}^{t}\left\|\Delta z_{\mu, \sigma}(s)\right\|_{L^{2}(\Omega)}^{2} d s
$$

Now, using Gronwall's inequality, we conclude that

$$
E_{\mu, \sigma}(t) \leq E_{\mu, \sigma}(0) \exp (C T)
$$

for $t \in[0, T]$.

From (8.30) and Lemma 8.1, we conclude that there exists a function $u$ such that, for all $T>0$, we have

$$
\begin{array}{cll}
u_{\mu} \longrightarrow u & \text { in } & C([0, T] ; V) \hookrightarrow L^{2}\left(0, T ; L^{2}(\Omega)\right), \\
u_{\mu}^{\prime} \longrightarrow u^{\prime} & \text { in } & L^{2}\left(0, T ; L^{2}\left(\Gamma_{1}\right)\right), \\
\Delta u_{\mu} \longrightarrow \Delta u & \text { in } & L^{2}\left(0, T ; L^{2}(\Omega)\right) .
\end{array}
$$

Moreover, from (7.22), (8.45) and (8.47), we have

$$
u_{\mu} \longrightarrow u \quad \text { in } \quad L^{2}\left(0, T ; H^{2}(\Omega)\right) .
$$

Considering the last convergence and those given by (8.45)-(8.47), it follows that $u$ is a weak solution in the sense of Definition 2.1.

Now, let $u_{1}$ and $u_{2}$ be two solutions of (1.1). Then $w=u_{1}-u_{2}$ satisfies

$$
\begin{cases}w_{t}-(\lambda+i \alpha) \Delta w+(\kappa+i \beta)\left(\left|u_{1}\right|^{p-1} u_{1}-\left|u_{2}\right|^{p-1} u_{2}\right)-\gamma w=0 & \text { in } \Omega \times \mathbb{R}_{+}, \\ \frac{\partial w}{\partial \nu}=-w_{t} & \text { on } \Gamma_{1} \times \mathbb{R}_{+}, \\ w=0 & \text { on } \Gamma_{0} \times \mathbb{R}_{+}, \\ w(0)=0 & \text { in } \Omega .\end{cases}
$$

Since $w \in H^{2}(\Omega)$ and $w_{t} \in L^{2}(\Omega)$, a. e. $t \in[0, T]$, we can repeat the procedure used in Lemma 8.1 with which we can get $w=0$ for a.e. $(x, t) \in \Omega \times[0, T]$.

\section{Inviscid limits}

In this section, we will prove Theorems 2.8 and 2.9. which state that the solutions of the CGLE subject to dynamic boundary conditions converge to the solution of NLS subject to same type of boundary conditions as the parameter pair $\epsilon=(\lambda, \kappa)$ tends to zero. 
We know from Lemma 7.1 that $u_{\epsilon}$ is uniformly bounded in $L^{\infty}(0, T ; V), \partial_{t} u_{\epsilon}$ is bounded in $L^{2}\left(0, T ; L^{2}\left(\Gamma_{1}\right)\right)$, and $\left|u_{\epsilon}\right|^{p-1} u_{\epsilon}$ is uniformly bounded in $L^{\infty}\left(0, T ; L^{(p+1)^{\prime}}(\Omega)\right)$. Using the main equation and Lemma 7.1 again, we can also see that $\partial_{t} u_{\epsilon}$ is uniformly bounded in $L^{2}\left(0, T ; V^{\prime}\right)$. From these bounds, it follows that there exists a subsequence of $u_{\epsilon}$ (denoted same) such that

$$
\begin{aligned}
u_{\epsilon} \rightarrow u & \text { weakly-star in } L^{\infty}(0, T ; V) ; \\
\partial_{t} u_{\epsilon} \rightarrow u_{t} & \text { weakly in } L^{2}\left(0, T ; L^{2}\left(\Gamma_{1}\right)\right) ; \\
\partial_{t} u_{\epsilon} \rightarrow u_{t} & \text { weakly in } L^{2}\left(0, T ; V^{\prime}\right) ; \\
\left|u_{\epsilon}\right|^{p-1} u_{\epsilon} \rightarrow \xi & \text { weakly-star in } L^{\infty}\left(0, T ; L^{(p+1)^{\prime}}(\Omega)\right) .
\end{aligned}
$$

Recall that the bounded sets in $X \equiv\left\{u \in L^{2}(0, T ; V) \mid u_{t} \in L^{2}\left(0, T ; V^{\prime}\right)\right\}$ are relatively compact in $L^{2}\left(0, T ; L^{2}(\Omega)\right)$. Therefore, we can assume $u_{\epsilon} \rightarrow u$ strongly in $L^{2}\left(0, T ; L^{2}(\Omega)\right)$ and $u_{\epsilon} \rightarrow u$ a.e. in $(0, T) \times \Omega$. This implies that $\left|u_{\epsilon}\right|^{p-1} u_{\epsilon} \rightarrow|u|^{p-1} u$ a.e. in $(0, T) \times \Omega$. But we have $\left|u_{\epsilon}\right|^{p-1} u_{\epsilon}$ is bounded in $L^{\infty}\left(0, T ; L^{(p+1)^{\prime}}(\Omega)\right)$. Therefore $\xi \equiv|u|^{p-1} u$. Now, letting $\epsilon \rightarrow 0$, we see that $u$ solves the idbvp for the NLS. This completes the proof of Theorem 2.8 .

In order to prove Theorem 2.9, we will restrict our attention to only dimension $N=2, p=3$, and $\beta>0$, for which we know that there is a global strong solution for the NLS [34]. To this end, let $u_{\epsilon}$ be the global strong solution of (2.15) with initial datum $u_{\epsilon}(0)=u_{\epsilon}^{0}$ and $p=3$. Suppose $u$ is a global strong solution of the cubic defocusing nonlinear Schrödinger equation with dynamic boundary conditions below $(\alpha, \beta>0)$ :

$$
\begin{cases}u_{t}-i \alpha \Delta u+i \beta|u|^{2} u-\gamma u=0 & \text { in } \Omega \times(0, T), \\ \frac{\partial u}{\partial \nu}=-u_{t} & \text { on } \Gamma_{1} \times(0, T), \\ u=0 & \text { on } \Gamma_{0} \times(0, T), \\ u(0)=u_{0} & \text { in } \Omega .\end{cases}
$$

Moreover, let us suppose that $u_{\epsilon}^{0} \rightarrow u_{0}$ as $\epsilon \rightarrow 0$ in $V$.

Set $w=u_{\epsilon}-u$. Then, $w$ solves the following problem:

$$
\begin{cases}w_{t}-i \alpha \Delta w+i \beta\left(\left|u_{\epsilon}\right|^{2} u_{\epsilon}-|u|^{2} u\right)-\gamma w-\lambda \Delta u_{\epsilon}+\kappa\left|u_{\epsilon}\right|^{2} u_{\epsilon}=0 & \text { in } \Omega \times(0, T), \\ \frac{\partial w}{\partial \nu}=-w_{t} & \text { on } \Gamma_{1} \times(0, T), \\ w=0 & \text { on } \Gamma_{0} \times(0, T), \\ w(0)=w_{0}^{\epsilon} \equiv u_{\epsilon}^{0}-u_{0} & \text { in } \Omega .\end{cases}
$$

We multiply $(9.55)$ by $-\Delta \bar{w}$, integrate over $\Omega \times(0, t)$, and take the real parts:

$$
\begin{aligned}
& \frac{1}{2}\|\nabla w(t)\|_{L^{2}(\Omega)}^{2}+\int_{0}^{t}\left\|w_{t}\right\|_{L^{2}\left(\Gamma_{1}\right)}^{2} d s+\beta \operatorname{Im} \int_{0}^{t}\left(\left|u_{\epsilon}\right|^{2} u_{\epsilon}-|u|^{2} u, \Delta w\right)_{L^{2}(\Omega)} d s \\
& \quad-\gamma \int_{0}^{t}\|\nabla w\|_{L^{2}(\Omega)}^{2} d s+\gamma \int_{0}^{t} \operatorname{Re}\left(w, \partial_{\nu} w\right)_{L^{2}\left(\Gamma_{1}\right)} d s+\lambda \operatorname{Re} \int_{0}^{t}\left(\Delta u_{\epsilon}, \Delta w\right)_{L^{2}(\Omega)} d s \\
& \quad-\kappa \int_{0}^{t} \operatorname{Re}\left(\left|u_{\epsilon}\right|^{2} u_{\epsilon}, \Delta w\right)_{L^{2}(\Omega)} d s=\frac{1}{2}\left\|\nabla w_{0}^{\epsilon}\right\|_{L^{2}(\Omega)}^{2} .
\end{aligned}
$$

Using the boundary condition, we estimate

$$
\frac{\gamma}{2} \operatorname{Re}\left(w, \partial_{\nu} w\right)_{L^{2}\left(\Gamma_{1}\right)} \leq \frac{1}{2}\left\|w_{t}\right\|_{L^{2}\left(\Gamma_{1}\right)}^{2}+\frac{\gamma^{2}}{8}\|w\|_{L^{2}\left(\Gamma_{1}\right)}^{2} \leq \frac{1}{2}\left\|w_{t}\right\|_{L^{2}\left(\Gamma_{1}\right)}^{2}+\frac{C \gamma^{2}}{8}\|w\|_{V}^{2},
$$


where the last inequality follows from the trace theorem. On the other hand, we calculate

$$
\begin{aligned}
\beta \operatorname{Re} & \int_{0}^{t}\left(\left|u_{\epsilon}\right|^{2} u_{\epsilon}-|u|^{2} u, \Delta w\right)_{L^{2}(\Omega)} d s \\
= & -\beta \operatorname{Re} \int_{0}^{t}\left(\nabla\left(\left|u_{\epsilon}\right|^{2} u_{\epsilon}-|u|^{2} u\right), \nabla w\right)_{L^{2}(\Omega)} d s+\beta \operatorname{Re} \int_{0}^{t}\left(\left|u_{\epsilon}\right|^{2} u_{\epsilon}-|u|^{2} u, \frac{\partial w}{\partial \nu}\right)_{L^{2}\left(\Gamma_{1}\right)} d s \\
\leq & \beta \int_{0}^{t}\left\|\nabla\left(\left|u_{\epsilon}\right|^{2} u_{\epsilon}-|u|^{2} u\right)\right\|_{L^{2}(\Omega)}\|\nabla w\|_{L^{2}(\Omega)} d s+\frac{1}{2} \int_{0}^{t}\left\|w_{t}\right\|_{L^{2}\left(\Gamma_{1}\right)}^{2} d s \\
& +\frac{\beta^{2}}{2} \int_{0}^{t}\left\|\left|u_{\epsilon}\right|^{2} u_{\epsilon}-|u|^{2} u\right\|_{L^{2}\left(\Gamma_{1}\right)}^{2} d s .
\end{aligned}
$$

One can prove that (by slightly modifying the calculations in [32, Lemma 3.3]):

$$
\begin{aligned}
\| \nabla & \left(\left|u_{\epsilon}\right|^{2} u_{\epsilon}-|u|^{2} u\right)\left\|_{L^{2}(\Omega)} \leq C\right\| u_{\epsilon}-u \|_{L^{2}(\Omega)}\left(\left\|u_{\epsilon}\right\|_{\infty}+\|u\|_{\infty}\right)^{2} \\
& +C\left\|u_{\epsilon}-u\right\|_{4}\left(\left\|u_{\epsilon}\right\|_{\infty}+\|u\|_{\infty}\right)\left(\left\|\nabla u_{\epsilon}\right\|_{4}+\|\nabla u\|_{4}\right) \\
\leq & C\left\|u_{\epsilon}-u\right\|_{V}\left(\left\|u_{\epsilon}\right\|_{H^{2}(\Omega)}+\|u\|_{H^{2}(\Omega)}\right)^{2} \\
& \quad+C\left\|u_{\epsilon}-u\right\|_{V}\left(\left\|u_{\epsilon}\right\|_{H^{2}(\Omega)}+\|u\|_{H^{2}(\Omega)}\right)\left(\left\|u_{\epsilon}\right\|_{H^{2}(\Omega)}^{\frac{1}{2}}\left\|u_{\epsilon}\right\|_{V}^{\frac{1}{2}}+\|u\|_{H^{2}(\Omega)}^{\frac{1}{2}}\|u\|_{V}^{\frac{1}{2}}\right) \\
\leq & C\|w\|_{V},
\end{aligned}
$$

where the second inequality above follows from the facts $V \hookrightarrow L^{2}(\Omega), H^{2}(\Omega) \hookrightarrow L^{\infty}(\Omega)(N=2)$, $H^{1}(\Omega) \hookrightarrow L^{4}(\Omega)(N=2)$, and the Gagliardo-Nirenberg inequality (7.12). Finally, the last inequality follows from the fact that $u_{\epsilon}, u \in C\left([0, T] ; V \cap H^{2}(\Omega)\right)$.

By using the same technique in [32, Lemma 3.3]), we have also the estimate

$$
\begin{aligned}
& \frac{\beta^{2}}{2}\left\|\left|u_{\epsilon}\right|^{2} u_{\epsilon}-|u|^{2} u\right\|_{L^{2}\left(\Gamma_{1}\right)}^{2} \\
& \quad \leq C\left(\left\|u_{\epsilon}\right\|_{L^{\infty}\left(\Gamma_{1}\right)}^{4}+\|u\|_{L^{\infty}\left(\Gamma_{1}\right)}^{4}\right)\left\|u_{\epsilon}-u\right\|_{L^{2}\left(\Gamma_{1}\right)}^{2} \\
& \quad \leq C\left(\left\|u_{\epsilon}\right\|_{H^{1}\left(\Gamma_{1}\right)}^{4}+\|u\|_{H^{1}\left(\Gamma_{1}\right)}^{4}\right)\left\|u_{\epsilon}-u\right\|_{L^{2}\left(\Gamma_{1}\right)}^{2} \leq C\left(\left\|u_{\epsilon}\right\|_{H^{\frac{3}{2}(\Omega)}}^{4}+\|u\|_{H^{\frac{3}{2}(\Omega)}}^{4}\right)\left\|u_{\epsilon}-u\right\|_{L^{2}\left(\Gamma_{1}\right)}^{2} \\
& \quad \leq C\left(\left\|u_{\epsilon}\right\|_{H^{2}(\Omega)}^{4}+\|u\|_{H^{2}(\Omega)}^{4}\right)\left\|u_{\epsilon}-u\right\|_{L^{2}\left(\Gamma_{1}\right)}^{2} \leq C\|w\|_{V}^{2},
\end{aligned}
$$

where the second inequality follows from the embedding $H^{1}\left(\Gamma_{1}\right) \hookrightarrow L^{\infty}\left(\Gamma_{1}\right)\left(\Gamma_{1}\right.$ is 1-dimensional manifold), the third inequality follows from the Sobolev trace theorem, and the last inequality follows from the fact that $u, u_{\epsilon} \in C\left([0, T] ; H^{2}(\Omega)\right)$ and the trace theorem.

Combining (9.56)-(9.60), it follows that

$$
\begin{aligned}
\frac{1}{2}\|\nabla w(t)\|_{L^{2}(\Omega)}^{2} \leq & \frac{1}{2}\left\|\nabla w_{0}^{\epsilon}\right\|_{L^{2}(\Omega)}^{2}+C \int_{0}^{T}\|w(t)\|_{V}^{2} d t \\
& +\lambda\left\|\Delta u_{\epsilon}\right\|_{L^{2}(\Omega)}\|\Delta w\|_{L^{2}(\Omega)}+\kappa\left\|u_{\epsilon}\right\|_{L^{6}(\Omega)}^{3}\|\Delta w\|_{L^{2}(\Omega)} d s .
\end{aligned}
$$

In the above inequality $\left\|u_{\epsilon}\right\|_{L^{6}(\Omega)},\|\Delta w\|_{L^{2}(\Omega)}$, and $\left\|\Delta u_{\epsilon}\right\|_{L^{2}(\Omega)}$ are bounded by a constant since $H^{1}(\Omega) \hookrightarrow L^{6}(\Omega)$, and $u_{\epsilon}, u \in C\left([0, T] ; H^{2}(\Omega)\right)$. Hence, (9.61) gives

$$
\|\nabla w(t)\|_{L^{2}(\Omega)}^{2} \leq\left\|u_{\epsilon}^{0}-u_{0}\right\|_{L^{2}(\Omega)}^{2}+(\lambda+\kappa) C+C \int_{0}^{T}\|w(t)\|_{V}^{2} d t .
$$

Unleashing the Gronwall's inequality, we have

$$
\left\|u_{\epsilon}-u\right\|_{V}^{2} \leq\left\|u_{\epsilon}^{0}-u_{0}\right\|_{L^{2}(\Omega)}^{2}+(\lambda+\kappa) C \exp (C T) .
$$


Hence, we have just proven that

$$
\left\|u_{\epsilon}-u\right\|_{V}=O(\lambda)+O(\kappa)
$$

as $\epsilon=(\lambda, \kappa) \rightarrow 0$.

\section{Long-time behavior of solutions}

In this section, we give the proofs of Theorem 2.10 and 2.11. We will first prove the easy case $(\gamma<0)$ and then prove the more subtle case $\gamma=0$. We know from the theory of weak solutions that for any

$$
u_{0} \in Q \equiv\left\{\varphi \in V \cap L^{p+1}(\Omega) \text { such that } \gamma_{0} \varphi \in L^{p+1}\left(\Gamma_{1}\right)\right\},
$$

there corresponds a unique global weak solution $u$ which solves (2.15). We claim that this solution tends to zero in $H^{1}(\Omega) \cap L^{p+1}(\Omega)$ sense as $t \rightarrow \infty$ with an exponential rate of decay. We will use the multiplier technique to prove this where the case $\gamma=0$ requires a special multiplier.

Case $1(\gamma<0)$ :

We consider the functional

$$
F(t) \equiv \frac{\alpha}{2}\|\nabla u(t)\|_{L^{2}(\Omega)}^{2}+\frac{\beta}{p+1}\|u(t)\|_{L^{p+1}(\Omega)}^{p+1} .
$$

Note that $F(t) \leq E(t)$ given in (7.2). Moreover, we have from (7.11) that

$$
F(t) \leq E(0)+\gamma \int_{0}^{t} F(s) d s
$$

Employing Gronwall's inequality:

$$
F(t) \leq E_{0} e^{-|\gamma| t}
$$

for $t \geq 0$, where

$$
\begin{aligned}
E_{0}= & E(0)=\frac{\alpha}{2}\left\|\nabla u_{0}\right\|_{L^{2}(\Omega)}^{2}+\frac{\beta}{p+1}\left\|u_{0}\right\|_{L^{p+1}(\Omega)}^{p+1}-\frac{\alpha \gamma}{2}\left\|u_{0}\right\|_{L^{2}\left(\Gamma_{1}\right)}^{2} \\
& +\frac{1}{p+1}(\alpha \kappa+\beta \lambda)\left\|u_{0}\right\|_{L^{p+1}\left(\Gamma_{1}\right)}^{p+1} \geq 0 .
\end{aligned}
$$

This proves that solutions decay in $H^{1}(\Omega) \cap L^{p+1}(\Omega)$ sense to zero at an exponential rate.

Case $2(\gamma=0)$ :

This case is more subtle and requires some control theoretic tools. We know that solutions of the CGLE with periodic or homogeneous Dirichlet/Neumann boundary conditions do not have to decay if $\gamma \geq 0$. We will prove that the dynamic boundary condition plays the role of a damping term and actually stabilizes the system from the boundary in the absence of an internal damping mechanism. Therefore, the boundary condition $\left.\frac{\partial u}{\partial \nu}\right|_{\Gamma_{1}}=-u_{t}$ can be considered a stabilizing boundary feedback within this context.

Note that Lemma 7.1 tells us that when $\gamma=0$, the energy is non-increasing. In order to prove stabilization, we will use an integral inequality given in the following lemma.

Lemma 10.1 ([48, Theorem 8.1]). Let $F: \mathbb{R}_{+} \rightarrow \mathbb{R}_{+}$be a non-increasing function and assume that there exists a constant $C>0$ such that

$$
\int_{t}^{\infty} F(s) d s \leq C F(t) \text { for all } t \geq 0 .
$$

Then,

$$
F(t) \leq F(0) e^{1-\frac{t}{C}} \text { for all } t \geq 0 \text {. }
$$


In order to obtain an integral inequality in the form of (10.66), we calculate

$$
\frac{d}{d t}(u, q \cdot \nabla u)_{L^{2}(\Omega)},
$$

which is by now a classical multiplier used in the control theory of PDEs and well-posedness theory of nonhomogeneous initial-boundary value problems. In (10.67), $q$ denotes a sufficiently smooth vector field on $\Omega$, which will be chosen in a special way later. We have the following lemma:

Lemma 10.2. Let $u$ be a global weak solution of $(2.15)$ and $q \in\left[C^{2}(\bar{\Omega})\right]^{n}$ be a real vector field over $\Omega$. Then, the following identity holds true:

$$
\begin{aligned}
& \frac{d}{d t}(u, q \cdot \nabla u)_{L^{2}(\Omega)}-\int_{\Gamma}(q \cdot \nu) u \bar{u}_{t} d \Gamma-(\kappa-i \beta) \int_{\Omega} \operatorname{div}(q)|u|^{p+1} d x \\
& \quad(\lambda-i \alpha) \int_{\Gamma} \operatorname{div}(q) u \partial_{\nu} \bar{u} d \Gamma-\lambda \int_{\Omega}\left(\left(\nabla(\operatorname{div}(q) \cdot \nabla \bar{u}) u+\operatorname{div}(q)|\nabla u|^{2}\right)\right) d x \\
& =2 i \lambda \operatorname{Im}(\Delta u, q \cdot \nabla u)-2 i \kappa \operatorname{Im}\left(|u|^{p-1} u, q \cdot \nabla u\right)+2 i \alpha \int_{\Gamma}\left(\partial_{\nu} u\right)(q \cdot \nabla \bar{u}) d \Gamma \\
& \quad-2 i \alpha \operatorname{Re} \sum_{m, j=1}^{n}\left(\left(\partial_{x_{m}} q_{j}\right) u_{x_{m}}, u_{x_{j}}\right)-i \alpha \int_{\Gamma}(q \cdot \nu)|\nabla u|^{2} d \Gamma \\
& -\frac{2}{p+1} i \beta \int_{\Gamma}(q \cdot n)|u|^{p+1} d \Gamma+\frac{2}{p+1} i \beta \int_{\Omega} \operatorname{div}(q)|u|^{p+1} d x .
\end{aligned}
$$

Proof. Since the proof can be made by slightly modifying the proof of [16, Lemma 2.1] and is based on integration by parts and tedious calculations. It is omitted here.

Let $q=x-x_{0}$. Then, by the given geometric assumption on the boundary of $\Omega$ in Theorem 2.11 we have $q \cdot \nu>0$ on $\Gamma_{1}$ and $q \cdot \nu \leq 0$ on $\Gamma_{0}$. We can also simply calculate $\operatorname{div}(q)=N$. It is important to notice that since $\left.u\right|_{\Gamma_{0}} \equiv 0$, one has $\left.\nabla u\right|_{\Gamma_{0}}=\left(\partial_{\nu} u\right) \nu$. Now, using these facts and Lemma 10.2, it follows that

$$
\begin{aligned}
& 2 \alpha \int_{t}^{T}\|\nabla u\|_{L^{2}(\Omega)}^{2} d t+\frac{N \beta(p-1)}{p+1} \int_{t}^{T}\|u\|_{L^{p+1}(\Omega)}^{p+1} d t \leq C\left(\|u(T)\|_{V}^{2}+\|u(t)\|_{V}^{2}\right)+\epsilon \int_{t}^{T}\|u\|_{V}^{2} d t \\
& \quad+C_{\epsilon} \int_{t}^{T}\left(\left\|u_{t}\right\|_{L^{2}\left(\Gamma_{1}\right)}^{2} d t+\|\Delta u\|_{L^{2}(\Omega)}^{2}+\|u\|_{L^{2 p}(\Omega)}^{2 p}\right) .
\end{aligned}
$$

But from Lemma 7.1, we know that

$$
\int_{t}^{T}\left(\left\|u_{t}\right\|_{L^{2}\left(\Gamma_{1}\right)}^{2} d t+\|\Delta u\|_{L^{2}(\Omega)}^{2}+\|u\|_{L^{2 p}(\Omega)}^{2 p}\right) \leq C(F(t)-F(T)) .
$$

Combining the last inequality with (10.69), it follows that

$$
\int_{t}^{T} F(t) d t \leq C F(T)+C(F(t)-F(T)) \leq C F(t),
$$

and hence

$$
\int_{t}^{\infty} F(t) d t \leq C F(t)
$$

for $t \geq 0$. Now, by using Lemma 10.1, exponential decay follows, and the proof of Theorem 2.11 is completed.

\section{Acknowledgment}

Türker Özsarı's research was supported by TÜBİTAK 3501 Career Grant \#115F055. 


\section{References}

[1] I.S. Aranson, L. Kramer, The world of the complex Ginzburg-Landau equation, Rev. Modern Phys. 74 (1) (2002) 99-143.

[2] P. Bechouche, A. Jüngel, Inviscid limits of the complex Ginzburg-Landau equation, Comm. Math. Phys. 214 (1) (2000) 201-226.

[3] T. Cazenave, F. Dickstein, F.B. Weissler, Finite-time blow-up for a complex Ginzburg-Landau equation, SIAM J. Math. Anal. 45 (1) (2013) 244-266.

[4] Philippe Clément, Noboru Okazawa, Motohiro Sobajima, Tomomi Yokota, A simple approach to the Cauchy problem for complex Ginzburg-Landau equations by compactness methods, J. Differential Equations 253 (4) (2012) 1250-1263.

[5] Charles R. Doering, John D. Gibbon, C. David Levermore, Weak and strong solutions of the complex Ginzburg-Landau equation, Physica D 71 (3) (1994) 285-318.

[6] J. Ginibre, G. Velo, The Cauchy problem in local spaces for the complex Ginzburg-Landau equation. I. Compactness methods, Physica D 95 (3-4) (1996) 191-228.

[7] J. Ginibre, G. Velo, The Cauchy problem in local spaces for the complex Ginzburg-Landau equation. II. Contraction methods, Comm. Math. Phys. 187 (1) (1997) 45-79.

[8] N. Masmoudi, H. Zaag, Blow-up profile for the complex Ginzburg-Landau equation, J. Funct. Anal. 255 (7) (2008) 1613-1666.

[9] Noboru Okazawa, Smoothing effect and strong L2-wellposedness in the complex Ginzburg-Landau equation, in: Differential Equations: Inverse and Direct Problems, in: Lect. Notes Pure Appl. Math., vol. 251, Chapman \& Hall/CRC, Boca Raton, FL, 2006, pp. 265-288.

[10] Noboru Okazawa, Tomomi Yokota, Monotonicity method applied to the complex Ginzburg-Landau and related equations, J. Math. Anal. Appl. 267 (1) (2002) 247-263.

[11] Daisuke Shimotsuma, Tomomi Yokota, Kentarou Yoshii, Existence and decay estimates of solutions to complex GinzburgLandau type equations, J. Differential Equations 260 (3) (2016) 3119-3149.

[12] Daisuke Shimotsuma, Tomomi Yokota, Kentarou Yoshii, Cauchy problem for the complex Ginzburg-Landau type equation with $L^{p}$-initial data, Math. Bohem. 139 (2) (2014) 353-361.

[13] Qi Tang, S. Wang, Time dependent Ginzburg-Landau equations of superconductivity, Physica D 88 (3-4) (1995) 139-166.

[14] Ole Morten Aamo, Andrey Smyshlyaev, Miroslav Krstić, Boundary control of the linearized Ginzburg-Landau model of vortex shedding, SIAM J. Control Optim. 43 (6) (2005) 1953-1971.

[15] Ole Morten Aamo, Andrey Smyshlyaev, Miroslav Krstić, Bjarne A. Foss, Output feedback boundary control of a GinzburgLandau model of vortex shedding, IEEE Trans. Automat. Control 52 (4) (2007) 742-748.

[16] Hongjun Gao, Charles Bu, Dirichlet inhomogeneous boundary value problem for the $\mathrm{n}+1$ complex Ginzburg-Landau equation, J. Differential Equations 198 (1) (2004) 176-195.

[17] Hongjun Gao, Xiaohua Gu, Charles Bu, A Neumann boundary value problem for a generalized Ginzburg-Landau equation Appl. Math. Comput. 134 (2-3) (2003) 553-560.

[18] Lionel Rosier, Bing-Yu Zhang, Null controllability of the complex Ginzburg-Landau equation, Ann. Inst. H. Poincaré Anal. Non Linéaire 26 (2) (2009) 649-673.

[19] Corentin Audiard, On the non-homogeneous boundary value problem for Schrödinger equations, Discrete Contin. Dyn. Syst. 33 (9) (2013) 3861-3884.

[20] Corentin Audiard, On the boundary value problem for the Schrödinger equation: compatibility conditions and global existence, Anal. PDE 8 (5) (2015) 1113-1143.

[21] A. Batal, T. Özsarı, Nonlinear Schrödinger equations on the half-line with nonlinear boundary conditions, Electron. J. Differential Equations (2016) 20 Paper No. 222.

[22] M.B. Erdoğan, N. Tzirakis, Regularity properties of the cubic nonlinear Schrödinger equation on the half line, J. Funct. Anal. 271 (9) (2016) 2539-2568.

[23] Athanassios S. Fokas, A. Alexandrou Himonas, Dionyssios Mantzavinos, The nonlinear Schrödinger equation on the halfline, Trans. Amer. Math. Soc. 369 (1) (2017) 681-709.

[24] Justin Holmer, The initial-boundary-value problem for the 1D nonlinear Schrödinger equation on the half-line, Differential Integral Equations 18 (6) (2005) 647-668.

[25] Elena I. Kaikina, Inhomogeneous Neumann initial-boundary value problem for the nonlinear Schrödinger equation, J. Differential Equations 255 (10) (2013) 3338-3356.

[26] Varga K. Kalantarov, Türker Özsar, Qualitative properties of solutions for nonlinear Schrödinger equations with nonlinear boundary conditions on the half-line, J. Math. Phys. 57 (2) (2016) 02151114 pp.

[27] I. Lasiecka, R. Triggiani, Optimal regularity, exact controllability and uniform stabilization of Schrödinger equations with Dirichlet control, Differential Integral Equations 5 (3) (1992) 521-535.

[28] Irena Lasiecka, Roberto Triggiani, Well-posedness and sharp uniform decay rates at the $L^{2}(\Omega)$-level of the Schrödinger equation with nonlinear boundary dissipation, J. Evol. Equ. 6 (3) (2006) 485-537.

[29] Türker Özsar, Varga K. Kalantarov, Irena Lasiecka, Uniform decay rates for the energy of weakly damped defocusing semilinear Schrödinger equations with inhomogeneous Dirichlet boundary control, J. Differential Equations 251 (7) (2011) $1841-1863$.

[30] Türker Özsar, Weakly-damped focusing nonlinear Schrödinger equations with Dirichlet control, J. Math. Anal. Appl 389 (1) (2012) 84-97.

[31] Türker Özsar, Global existence and open loop exponential stabilization of weak solutions for nonlinear Schrödinger equations with localized external Neumann manipulation, Nonlinear Anal. 80 (2013) 179-193. 
[32] Türker Özsar, Well-posedness for nonlinear Schrödinger equations with boundary forces in low dimensions by Strichartz estimates, J. Math. Anal. Appl. 424 (1) (2015) 487-508.

[33] Walter Strauss, Charles Bu, An inhomogeneous boundary value problem for nonlinear Schrödinger equations, J. Differential Equations 173 (1) (2001) 79-91.

[34] Marcelo M. Cavalcanti, Wellington J. Corrêa, Irena Lasiecka, Christopher Lefler, Well-posedness and uniform stability for nonlinear Schrödinger equations with dynamic/Wentzell boundary conditions, Indiana Univ. Math. J. 65 (5) (2016) 1445-1502.

[35] A.D. Ventcel', On boundary conditions for multi-dimensional diffusion processes, Theory Probab. Appl. 4 (1959) 164-177.

[36] Angelo Favini, Gisèle Ruiz Goldstein, Jerome A. Goldstein, Silvia Romanelli, The heat equation with generalized Wentzell boundary condition, J. Evol. Equ. 2 (1) (2002) 1-19.

[37] Marcelo M. Cavalcanti, Irena Lasiecka, Daniel Toundykov, Geometrically constrained stabilization of wave equations with Wentzell boundary conditions, Appl. Anal. 91 (8) (2012) 1427-1452.

[38] Angelo Favini, Giséle Ruiz Goldstein, Jerome A. Goldstein, Silvia Romanelli, $C_{0}$-semigroups generated by second order differential operators with general Wentzell boundary conditions, Proc. Amer. Math. Soc. 128 (7) (2000) 1981-1989.

[39] Angelo Favini, Giséle Ruiz Goldstein, Jerome A. Goldstein, Silvia Romanelli, The heat equation with generalized Wentzell boundary condition, J. Evol. Equ. 2 (1) (2002) 1-19.

[40] Takayoshi Ogawa, Tomomi Yokota, Uniqueness and inviscid limits of solutions for the complex Ginzburg-Landau equation in a two-dimensional domain, Comm. Math. Phys. 245 (1) (2004) 105-121.

[41] Jiahong Wu, The inviscid limit of the complex Ginzburg-Landau equation, J. Differential Equations 142 (2) (1998) $413-433$.

[42] I. Lasiecka, D. Tataru, Uniform boundary stabilization of semilinear wave equations with nonlinear boundary damping, Differential Integral Equations 6 (3) (1993) 507-533.

[43] H. Brézis, Functional Analysis, Sobolev Spaces and Partial Differential Equations, in: Universitext, Springer, New York, 2011.

[44] R.E. Showalter, Monotone Operators in Banach Space and Nonlinear Partial Differential Equations, in: Mathematical Surveys and Monographs, vol. 49, American Mathematical Society, Providence, RI, 1997.

[45] J.-L. Lions, E. Magenes, Problèmes aux Limites Non Homogènes et Applications. Vol. 1, in: Travaux et Recherches Mathématiques, No. 17, Dunod, Paris, 1968.

[46] A. Pazy, Semigroups of Linear Operators and Applications to Partial Differential Equations, in: Applied Mathematical Sciences, vol. 44, Springer-Verlag, New York, 1983.

[47] H. Brézis, T. Gallouet, Nonlinear Schrödinger evolution equations, Nonlinear Anal. 4 (4) (1980) 677-681.

[48] V. Komornik, Exact controllability and stabilization, in: The Multiplier Method, in: RAM: Research in Applied Mathematics, John Wiley \& Sons, Ltd, Masson, Paris, Chichester, 1994. 